ESAIM: COCV 27 (2021) 89

https://doi.org/10.1051/cocv/2021085
ESAIM: Control, Optimisation and Calculus of Variations

www.esaim-cocv.org

\title{
MEAN FIELD APPROACH TO STOCHASTIC CONTROL WITH PARTIAL INFORMATION
}

\author{
Alain Bensoussan ${ }^{1,2, *}$ And Sheung Chi Phillip YAM $^{3, * *, * * *}$ (1)
}

\begin{abstract}
In our present article, we follow our way of developing mean field type control theory in our earlier works [Bensoussan et al., Mean Field Games and Mean Field Type Control Theory. Springer, New York (2013)], by first introducing the Bellman and then master equations, the system of HamiltonJacobi-Bellman (HJB) and Fokker-Planck (FP) equations, and then tackling them by looking for the semi-explicit solution for the linear quadratic case, especially with an arbitrary initial distribution; such a problem, being left open for long, has not been specifically dealt with in the earlier literature, such as Bensoussan [Stochastic Control of Partially Observable Systems. Cambridge University Press, (1992)] and Nisio [Stochastic control theory: Dynamic programming principle. Springer (2014)], which only tackled the linear quadratic setting with Gaussian initial distributions. Thanks to the effective meanfield theory, we propose a solution to this long standing problem of the general non-Gaussian case. Besides, our problem considered here can be reduced to the model in Bandini et al. [Stochastic Process. Appl. 129 (2019) 674-711], which is fundamentally different from our present proposed framework.
\end{abstract}

Mathematics Subject Classification. 49N30, 49N70, 49N90, 60H15, 60H30, 91A16.

Received December 23, 2020. Accepted August 4, 2021.

Dedicated to Enrique Zuazua for his 60th birthday

\section{INTRODUCTION}

With the development of nonlinear filtering, stochastic control with partial information has been one of the most important and well established topics in control theory, where the underlying controlled state dynamics is also influenced by unobservable uncertainties. With full information, the optimal control can often be taken in a Markovian feedback form as a function in the state up to the present time. In contrast, under partial information, the state of the system is unobservable, yet Markov property can still be reinstated by introducing

* Research supported by grant from the National Science Foundation 1905449.

** Phillip Yam acknowledges the financial supports from HKGRF-14300717 with the project title "New kinds of Forward-backward Stochastic Systems with Applications", HKGRF-14300319 with the project title "Shape-constrained Inference: Testing for Monotonicity", and HKGRF-14301321 with the project title "General Theory for Infinite Dimensional Stochastic Control: Mean Field and Some Classical Problems". He also thanks Columbia University for the kind invitation to be a visiting faculty member in the Department of Statistics during his sabbatical leave. He also recalled the unforgettable moments and the happiness shared with his beloved father and used this work in memory of his father's brave battle against liver cancer.

Keywords and phrases: Duncan-Mortensen-Zakai equations, mean field type control problem, Bellman and Master equations, filtering formulae with non-Gaussian initial conditions, linear dynamics and quadratic payoff, settings with Gaussian or nonGaussian initial distributions, Riccati equations.

${ }^{1}$ International Center for Decision and Risk Analysis, Jindal School of Management, University of Texas at Dallas.

2 School of Data Science, The City University of Hong Kong.

3 Department of Statistics, The Chinese University of Hong Kong.

*** Corresponding author: scpyam@sta.cuhk.edu.hk

(C) The authors. Published by EDP Sciences, SMAI 2021 
the conditional probability, of the controlled dynamics, given the available observation; which is the state of an infinite-dimensional dynamic system satisfying Kushner equation. The classical stochastic control problem under partial information, as, for instance, described in the books of Bensoussan [3] and Nisio [13], can be formulated as a control problem for the (controlled) Zakai equation, whose solution is the unnormalized conditional probability distribution of the state of the system, which is not directly accessible. It is however noteworthy that [3] requires that, even under the linear quadratic setting, the initial distribution should be Gaussian, which is fairly restrictive.

Conventionally, two approaches are commonly adopted in order to solve the stochastic control problem under partial observation. The first one is through the stochastic Pontryagin Maximum Principle which describes necessary conditions for the optimality in terms of the dynamics and the corresponding adjoint process, see e.g. [3] and [17]. The second one is via Dynamic Programming Principle under the assumption that the conditional expectation with respect to the observation process admits a density, then one obtains the controlled Zakai equation, also known as the Duncan-Mortensen-Zakai equation, or Zakai equation in short in the rest of this article, for the proxy of unnormalised conditional probability density, which leads to the fully nonlinear HJB equation, see $[10,11]$ and references therein. There are also more relevant developments recently, see for instance [8] that provides a discrete setting, and [16] discussing the applications in machine learning; for other works that are more related to mean field theory and partially observable systems, please refer to $[1,5,6,9,14,15]$, and references therein. Particularly, another notable recent work is [13], where the linear quadratic problem is also dealt with when the initial distribution is Gaussian as in [3], yet the problem with an arbitrary initial distribution has not been tackled until our present work; furthermore, [13] does not state the separation principle, nor does it exploit the filtering formulae with non-Gaussian initial conditions as discussed in [12].

By introducing the unnormalized conditional probability, the solution of the controlled Zakai equation which is a stochastic Fokker-Planck equation, the classical stochastic control problem under partial information can be formulated as a control problem for the Zakai equation. This new mathematical problem can be solved very similarly to those commonly encountered in Mean Field Type Control theory, and this newly developed method is introduced here as the first time in the literature of filtering. Moreover, to the best of our knowledge, even for the setting with a linear dynamics and quadratic payoff, the filtering problem with non-Gaussian initial distributions has been standing for long, and we shall illustrate the power of our proposed mean-field approach and resolve this open problem for the linear quadratic case here.

Mean Field Type Control theory is much later than the development of Stochastic Control with partial information, the tools, techniques and concepts obtained in the last decade, for Mean Field Games and Mean Field Type Control, have not been used for the control of Zakai equation. It is the objective of this work to connect these two theories; indeed, there is a notable difference, namely, we here deal with unnormalized probabilities instead of proper probabilities like those dealt in the canonical problems in Mean Field Theory. Motivated by our former work in [4], particularly by introducing Bellman equations, master equations, and the system of forward-backward equations, we aim to establish and then study these equations to the greatest extent in this paper. Besides grasping the power of new tools, we also gain new insights for the problem of stochastic control with partial information. For Mean Field Theory, we get new interesting applications, but also new problems. The possibility of using direct methods is, of course, quite fruitful; indeed, if Mean Field Type Control Theory is a very comprehensive and powerful framework, it leads to very complex equations, like Master equation, which is a nonlinear infinite dimensional PDE, for which general theorems are hardly available, although an active research in this direction has been conducted, see Cardaliaguet et al. [7]. Direct methods are particularly useful to obtain regularity results. We shall develop in detail the linear quadratic regulator problem, but because we cannot just consider the Gaussian case, well-known results, like the separation principle are not available. An interesting and important result is available in the literature, due to A. Makowski, [12]. It describes the solution of Zakai equation for linear systems especially with general initial condition, i.e. non-Gaussian setting. As mentioned, this result has not been exploited for the control aspect in the literature before our work. We show that the separation principle can be extended for quadratic pay-off functionals, but the Kalman filter is much more complex than in the case with Gaussian initial distributions. We finally discuss in the last section the recent approach of Bandini et al. [2] for comparison with our approach. Their approach is very 
fruitful for the general theory, but does not lead to the explicit solution as we obtain in this paper. Roughly speaking, our problem considered here can be reduced to that investigated in an interesting recent alternative formulation in [2], which is fundamentally different from our present proposed framework; indeed, their set up leads to a conditional probability (hence normalized) solution of a linear stochastic PDE, which is another special case of Duncan-Mortensen-Zakai equations but different from the one we obtained here. They have also provided an example with linear equations, which we believe does not cover the proposed linear quadratic case, with an arbitrary initial distribution, as extensively dealt in the present work; particularly our system remains nonlinear in the framework of [2], which is due to the complication of their approach.

The organization of this article is as follows. Section 2 formulates the stochastic control problem with partial information, and introduces the corresponding controlled Zakai equation. The key toolkit of mean field approach is introduced in Section 3, and then we attempt to obtain a weak solution of the stochastic control problem by solving the Zakai equation under a weak formulation in Section 4. In Section 5, we present the solution of the linear quadratic problem with an arbitrary initial distribution using the mean field approach, and hence demonstrate the effecitiveness of the mean-field theory. Finally in Section 6 , we compare our approach with the recent one in [2].

\section{Stochastic CONTROL With PARTial INFORMATiON}

\subsection{The problem}

We first describe the problem formally, without making precise the assumptions. The state of the system $x(t) \in \mathbb{R}^{n}$ is a solution of a diffusion:

$$
\left\{\begin{aligned}
\mathrm{d} x & =g(x, v) \mathrm{d} t+\sigma(x) \mathrm{d} w \\
x(0) & =\xi
\end{aligned}\right.
$$

and we assume that there exists a probability space $(\Omega, \mathcal{A}, \mathbb{P})$ on which a random variable $\xi$ and a standard $\mathbb{R}^{n}$ -valued Wiener process, which is independent of $\xi$, are constructed. There is a control $v(t)$ in the drift term, with values in $\mathbb{R}^{m}$. Since, we cannot access to the state $x(t)$, which is not observable, it can neither be defined by a feedback on the state, nor adapted to the state. Formally, we have an observation equation:

$$
\mathrm{d} z=h(x) \mathrm{d} t+\mathrm{d} b(t)
$$

in which $z(t)$, with values in $\mathbb{R}^{d}$, represents the observation and $b(t)$ is another Wiener process, independent of the pair $(\xi, w(\cdot))$. The function $h(x)$ corresponds to the measurement of the state $x$ and $b(t)$ captures a measurement error. So the control $v(t)$ should be adapted to the process $z(t)$, not a feedback of course. It is well-known that this construction is ill-posed. Indeed, the control is adapted to the observation, which henceforth depends also on the state, which depends on the control. It is a chicken and egg matter, that is usually solved by the Girsanov theorem, at the price of constructing appropriately the Wiener process $b(t)$. In practice, we construct on $(\Omega, \mathcal{A}, \mathbb{P})$ three objects: $\xi, w(\cdot)$ and $z(\cdot)$. The processes $w(\cdot)$ and $z(\cdot)$ are independent Wiener processes on $\mathbb{R}^{n}$ and $\mathbb{R}^{d}$, respectively, while $\xi$ is independent of these two processes. We set

$$
\mathcal{F}^{t}=\sigma(\xi, w(s), z(s), s \leq t), \quad \mathcal{Z}^{t}=\sigma(z(s), s \leq t),
$$

the filtrations on $(\Omega, \mathcal{A}, \mathbb{P})$ generated by $(\xi, w(\cdot), z(\cdot))$ and $z(\cdot)$ respectively. The process $z(\cdot)$ is the observation process, but it is defined exogeneously. We can then choose the control $v(\cdot)$ as a process with values in $\mathbb{R}^{m}$, which is adapted to the filtration $\mathcal{Z}^{t}$. So, it is perfectly well-defined, as well as the process $x(\cdot)$ as the solution of (2.1). In fact in (2.1) $v(\cdot)$ is fixed, like $\xi$ and $w(\cdot)$, and we assume that we can solve the SDE (2.1) in a strong sense. So $x(\cdot)$ is well-defined. Here comes Girsanov theorem. We define the scalar $\mathbb{P}, \mathcal{F}^{t}$-martingale $\eta(t)$, which 
is the solution of the equation:

$$
\left\{\begin{aligned}
\mathrm{d} \eta(t) & =\eta(t) h(x(t)) \cdot \mathrm{d} z(t) \\
\eta(0) & =1
\end{aligned}\right.
$$

This martingale allows to define a new probability on $\Omega, \mathcal{A}$, denoted by $\mathbb{P}^{v(\cdot)}$ to emphasize the fact that it depends on the control $v(\cdot)$. It is given by the Radon-Nikodym derivative:

$$
\left.\frac{\mathrm{d} \mathbb{P}^{v(\cdot)}}{\mathrm{d} \mathbb{P}}\right|_{\mathcal{F}^{t}}=\eta(t)
$$

Finally, we define the process

$$
b^{v(\cdot)}(t):=z(t)-\int_{0}^{t} h(x(s)) \mathrm{d} s,
$$

which also depends on the control decision. We take a finite horizon $T$ in the rest of this article. Making the change of probability from $\mathbb{P}$ to $\mathbb{P}^{v(\cdot)}$ and considering the probability space $\left(\Omega, \mathcal{F}^{T}, \mathbb{P}^{v(\cdot)}\right)$, then $b^{v(\cdot)}$ appears as a standard Wiener process, which is independent of $w(\cdot)$ and $\xi$. Therefore, (2.5) is a template of (2.2) as far as probability laws are concerned. We can then rigorously define the control problem (without the chicken and egg matter):

$$
J(v(\cdot))=\mathbb{E}^{v(\cdot)}\left[\int_{0}^{T} f(x(t), v(t)) \mathrm{d} t+f_{T}(x(T))\right]
$$

in which the functions $f(x, v)$ and $f_{T}(x)$ represent the running cost and the final cost, respectively, contributing to the payoff functional to be minimized. The notation $\mathbb{E}^{v(\cdot)}$ refers to the expected value with respect to the probability law $\mathbb{P}^{v(\cdot)}$.

Remark 2.1. The previous presentation, which is currently the common one to formalize stochastic control problems with partial information, has a slight drawback, in comparison with the description of the problem with full information. With full information, there is no $\mathcal{Z}^{t}$ and the underlying filtration $\mathcal{F}^{t}=\sigma(\xi, w(\cdot))$ is accessible. A control $v(\cdot)$ is a stochastic process adapted to $\mathcal{F}^{t}$. We call it open-loop, because it is externally defined (this should not be confused with the practice in engineering to call open-loop controls, those which are deterministic functions of time). But, since the state $x(t)$ is also accessible, we can also consider controls, defined by feedbacks built on the state. In spite of the difference in the definition, the class of feedback controls is contained in that of open-loop controls. Indeed, after constructing the trajectory corresponding to a feedback, we feed the feedback with that trajectory. We get an open-loop control, leading to the same cost. The interesting feature of cost functionals of the type (2.6) is that the optimal open-loop control is defined by a feedback. So restricting ourselves to the subclass of feedback controls does not hurt. This is very important, when we formulate the control problem in the framework of mean-field theory. In mean-field theory, we must define the control with a feedback. Surprisingly, open-loop controls and feedback controls will lead to different solutions. In the case of partial information, we have unfortunately no choice. There is no feedback, since the state is not accessible. With the formulation above, the observation filtration $\mathcal{Z}^{t}$ is externally defined, and the control is open-loop, since it is externally defined as a process adapted to $\mathcal{Z}^{t}$. It is important to have this discussion in mind, when we formulate the problem with mean-field theory. 


\subsection{Control of Zakai equation}

Note first that the functional (2.6) can be written as

$$
J(v(\cdot))=\mathbb{E}\left[\int_{0}^{T} \eta(t) f(x(t), v(t)) \mathrm{d} t+\eta(T) f_{T}(x(T))\right] .
$$

This is obtained by using the Radon-Nikodym derivative (2.4) and the martingale property of $\eta(t)$. We next recall the classical nonlinear filtering theory result. Let $\Psi(x)$ be any bounded continuous function. We want to express the conditional expectation $\mathbb{E}^{v(\cdot)}\left[\Psi(x(t)) \mid \mathcal{Z}^{t}\right]$ of the random variable $\Psi(x(t))$ with respect to the $\sigma$-algebra $\mathcal{Z}^{t}$, on the probability space $\left(\Omega, \mathcal{A}, \mathbb{P}^{v(\cdot)}\right)$. We have the basic result of non-linear filtering theory:

$$
\mathbb{E}^{v(\cdot)}\left[\Psi(x(t)) \mid \mathcal{Z}^{t}\right]=\frac{\mathbb{E}\left[\eta(t) \Psi(x(t)) \mid \mathcal{Z}^{t}\right]}{\mathbb{E}\left[\eta(t) \mid \mathcal{Z}^{t}\right]}=\frac{\int_{\mathbb{R}^{n}} \Psi(x) q(x, t) \mathrm{d} x}{\int_{\mathbb{R}^{n}} q(x, t) \mathrm{d} x},
$$

where $q(x, t)$ is called the unnormalized conditional probaility density of the random variable $x(t)$ with respect to the $\sigma$-algebra $\mathcal{Z}^{t}$. One can directly read off from (2.8) that the conditional probability density itself is given by $\frac{q(x, t)}{\int_{\mathbb{R}^{n}} q(\xi, t) \mathrm{d} \xi}$. The function $q(x, t)$ is a random field adapted to the filtration $\mathcal{Z}^{t}$. It is the solution of a stochastic PDE:

$$
\left\{\begin{array}{l}
\mathrm{d} q+A^{*} q(x, t) \mathrm{d} t+\operatorname{div}(g(x, v(t)) q(x, t)) \mathrm{d} t-q(x, t) h(x) \cdot \mathrm{d} z(t)=0, \\
q(x, 0)=q_{0}(x)
\end{array}\right.
$$

in which $A^{*}$ is the second order differential operator:

$$
A^{*} \varphi(x)=-\sum_{i, j=1}^{n} \frac{\partial^{2}}{\partial x_{i} \partial x_{j}}\left(a_{i j} \varphi(x)\right)
$$

which is the dual of

$$
A \varphi(x)=-\sum_{i, j=1}^{n} a_{i j}(x) \frac{\partial^{2} \varphi}{\partial x_{i} \partial x_{j}}
$$

with $a(x)=\frac{1}{2}\left(\sigma \sigma^{*}\right)(x)$. The initial condition $q_{0}(x)$ is the probability density of $\xi$. We suppose that $\xi$ has a probability density. The random field $q(x, t)$ depends on $v(\cdot)$ and is thus denoted by $q^{v(\cdot)}(x, t)$. From $(2.8)$ and (2.7), we can write the pay-off $J(v(\cdot))$ as

$$
J(v(\cdot))=\mathbb{E}\left[\int_{0}^{T} \int_{\mathbb{R}^{n}} q^{v(\cdot)}(x, t) f(x, v(t)) \mathrm{d} x \mathrm{~d} t+\int_{\mathbb{R}^{n}} q^{v(\cdot)}(x, T) f_{T}(x) \mathrm{d} x\right] .
$$

The minimization of $J(v(\cdot))$ is a stochastic control problem for a dynamic system whose evolution is governed by the stochastic PDE (2.9).

Remark 2.2. We can elaborate more on the difference between feedback controls and open-loop controls, as addressed in Remark 2.1, by considering equation (2.9) describing the evolution of the state $q(x, t)$. In this equation $v(t)$ is a stochastic process adapted to the filtration $\mathcal{Z}^{t}$, so it is fixed with respect to the space variable $x$. 


\section{MEAN FIELD APPROACH}

\subsection{Preliminaries}

We define the value function:

$$
\Phi\left(q_{0}, 0\right)=\inf _{v(\cdot)} J(v(\cdot))
$$

and following the main concept of Dynamic Programming, we embed this value function into a family parametrized by initial conditions $q$ and $t$, where $q$ denotes an unnormalized probability density on $\mathbb{R}^{n}$. We also make precise the choice of the functional space in which the function $q(x)$ lies. To fix ideas, we take $q \in L^{2}\left(\mathbb{R}^{n}\right) \cap L^{1}\left(\mathbb{R}^{n}\right)$ and $q(x) \geq 0$. We shall assume that

$$
\int_{\mathbb{R}^{n}}|x|^{2} q(x) \mathrm{d} x<+\infty .
$$

Considering functionals on $L^{2}\left(\mathbb{R}^{n}\right), \Psi(q)$, we say that it is Gâteaux differentiable, with Gâteaux derivative $\frac{\partial \Psi}{\partial q}(q)(x)$ if the function $t \mapsto \Psi(q+t \tilde{q})$ is differentiable with the following formula:

$$
\frac{d}{\mathrm{~d} t} \Psi(q+t \tilde{q})=\int_{\mathbb{R}^{n}} \frac{\partial \Psi}{\partial q}(q+t \tilde{q})(x) \tilde{q}(x) \mathrm{d} x, \forall \tilde{q}(\cdot) \in L^{2}\left(\mathbb{R}^{n}\right) \cap L^{1}\left(\mathbb{R}^{n}\right), \tilde{q}(x) \geq 0, t \geq 0 .
$$

We shall assume that with $(q, x) \mapsto \frac{\partial \Psi}{\partial q}(q)(x)$ is continuous, satisfying:

$$
\left|\frac{\partial \Psi}{\partial q}(q)(x)\right| \leq c(q)\left(1+|x|^{2}\right)
$$

such that $c(q)$ is continuous and bounded on any bounded subsets of $L^{2}\left(\mathbb{R}^{n}\right)$. We also need the concept of second order Gâteaux derivative. The second order Gâteaux derivative is a functional $\frac{\partial^{2} \Psi}{\partial q^{2}}(q)(\xi, \eta)$ such that the function $t \mapsto \Psi(q+t \tilde{q})$ is twice differentiable in $t$ so that

$$
\frac{d^{2}}{\mathrm{~d} t^{2}} \Psi(q+t \tilde{q})=\int_{\mathbb{R}^{n}} \int_{\mathbb{R}^{n}} \frac{\partial^{2} \Psi}{\partial q^{2}}(q+t \tilde{q})(\xi, \eta) \tilde{q}(\xi) \tilde{q}(\eta) \mathrm{d} \xi \mathrm{d} \eta
$$

Moreover, the function $(q, \xi, \eta) \mapsto \frac{\partial^{2} \Psi}{\partial q^{2}}(q)(\xi, \eta)$ is continuous satisfying:

$$
\left|\frac{\partial^{2} \Psi}{\partial q^{2}}(q)(\xi, \eta)\right| \leq c(q)\left(1+|\xi|^{2}+|\eta|^{2}\right)
$$

with $c(q)$ continuous and bounded on any bounded subsets of $L^{2}\left(\mathbb{R}^{n}\right)$. From formula (3.5), it is clear that we can choose $\frac{\partial^{2} \Psi}{\partial q^{2}}(q)(\xi, \eta)$ to be symmetric in $(\xi, \eta)$. Set $f(t)=\Psi(q+t \tilde{q})$. Then, combining the above assumptions (3.2), (3.4) and (3.6), we can assert that $f(t)$ is $C^{2}$. Therefore, we have the identity:

$$
f(1)=f(0)+f^{\prime}(0)+\int_{0}^{1} \int_{0}^{1} t f^{\prime \prime}(s t) \mathrm{d} s \mathrm{~d} t
$$


which leads to the formula:

$$
\Psi(q+\tilde{q})=\Psi(q)+\int_{\mathbb{R}^{n}} \frac{\partial \Psi}{\partial q}(q)(x) \tilde{q}(x) \mathrm{d} x+\int_{0}^{1} \int_{0}^{1} t \int_{\mathbb{R}^{n}} \int_{\mathbb{R}^{n}} \frac{\partial^{2} \Psi}{\partial q^{2}}(q+s t \tilde{q})(\xi, \eta) \tilde{q}(\xi) \tilde{q}(\eta) \mathrm{d} \xi \mathrm{d} \eta \mathrm{d} s \mathrm{~d} t
$$

\subsection{Bellman equation}

We consider the control problem with initial conditions $q(x)$ at time $t$, or $(q, t)$ for short:

$$
\left\{\begin{array}{l}
\mathrm{d} q+A^{*} q(x, s) \mathrm{d} s+\operatorname{div}(g(x, v(s)) q(x, s)) \mathrm{d} s-q(x, s) h(x) \cdot \mathrm{d} z(s)=0, s>t, \\
q(x, t)=q(x)
\end{array}\right.
$$

such that

$$
J_{q, t}(v(\cdot)):=\mathbb{E}\left[\int_{t}^{T} \int_{\mathbb{R}^{n}} q^{v(\cdot)}(x, s) f(x, v(s)) \mathrm{d} x \mathrm{~d} s+\int_{\mathbb{R}^{n}} q^{v(\cdot)}(x, T) f_{T}(x) \mathrm{d} x\right] .
$$

and define the value function:

$$
\Phi(q, t)=\inf _{v(\cdot)} J_{q, t}(v(\cdot))
$$

Assuming that the value function has derivatives $\frac{\partial \Phi}{\partial t}(q, t), \frac{\partial \Psi}{\partial q}(q)(x), \frac{\partial^{2} \Psi}{\partial q^{2}}(q)(\xi, \eta)$, then, by standard arguments, we can check formally that $\Phi(q, t)$ is the solution of the Bellman equation:

$$
\left\{\begin{array}{l}
\frac{\partial \Phi}{\partial t}-\int_{\mathbb{R}^{n}} A \frac{\partial \Phi}{\partial q}(q, t)(x) q(x) \mathrm{d} x+\frac{1}{2} \int_{\mathbb{R}^{n}} \int_{\mathbb{R}^{n}} \frac{\partial^{2} \Phi}{\partial q^{2}}(q, t)(\xi, \eta) q(\xi) q(\eta) h(\xi) \cdot h(\eta) \mathrm{d} \xi \mathrm{d} \eta \\
\quad+\inf _{v} \int_{\mathbb{R}^{n}} q(x)\left(f(x, v)+D_{x} \frac{\partial \Phi}{\partial q}(q, t)(x) \cdot g(x, v)\right) \mathrm{d} x=0, \\
\Phi(q, T)=\int_{\mathbb{R}^{n}} f_{T}(x) q(x) \mathrm{d} x .
\end{array}\right.
$$

The optimal open-loop control is obtained by achieving the infimum in (3.11). We derive a functional $\hat{v}(q, t)$, which is a feedback in $q$ but not in $x$. We can then feed the Zakai equation (3.8) with this feedback to get the optimal state equation:

$$
\left\{\begin{array}{l}
\mathrm{d} q+A^{*} q(x, s) \mathrm{d} s+\operatorname{div}(g(x, \hat{v}(q, s)) q(x, s)) \mathrm{d} s-q(x, s) h(x) \cdot \mathrm{d} z(s)=0, s>t, \\
q(x, t)=q(x)
\end{array}\right.
$$

Once we solve this stochastic PDE, we obtain the optimal state $\hat{q}(s):=\hat{q}(x, s)$. We then define the control $\hat{v}(s)=\hat{v}(\hat{q}(s), s)$, which is indeed adapted to the filtration $\mathcal{Z}_{t}^{s}=\sigma(z(\tau)-z(t), t \leq \tau \leq s)$. This is the optimal open-loop control. 


\subsection{The master equation}

The functional $\hat{v}(q, t)$ defined at the very end of the last subsection depends on the function $\frac{\partial \Phi}{\partial q}(q, t)(x)$ denoted by $U(x, q, t)$. So, it is convenient to denote by $\hat{v}(q, U)$ the vector $v$ which achieves the minimum of

$$
\inf _{v} \int_{\mathbb{R}^{n}} q(x)\left(f(x, v)+D_{x} U(x, q) \cdot g(x, v)\right) \mathrm{d} x,
$$

in which we omit to write explicity the arguement $t$ for simplicity. Bellman equation (3.11) can be rewritten as:

$$
\left\{\begin{array}{l}
\frac{\partial \Phi}{\partial t}-\int_{\mathbb{R}^{n}} A_{x} U(x, q) q(x) \mathrm{d} x+\frac{1}{2} \int_{\mathbb{R}^{n}} \int_{\mathbb{R}^{n}} \frac{\partial^{2} \Phi}{\partial q^{2}}(q, t)(\xi, \eta) q(\xi) q(\eta) h(\xi) \cdot h(\eta) \mathrm{d} \xi \mathrm{d} \eta \\
\quad+\int_{\mathbb{R}^{n}} q(x)\left(f(x, \hat{v}(q, U))+D_{x} U(x, q) \cdot g(x, \hat{v}(q, U))\right) \mathrm{d} x=0, \\
\Phi(q, T)=\int_{\mathbb{R}^{n}} f_{T}(x) q(x) \mathrm{d} x .
\end{array}\right.
$$

It is also convenient to set

$$
V(q, t)(x, y)=\frac{\partial^{2} \Phi}{\partial q^{2}}(q, t)(x, y)
$$

Therefore, Bellman equation reads

$$
\left\{\begin{array}{l}
\frac{\partial \Phi}{\partial t}-\int_{\mathbb{R}^{n}} A_{x} U(x, q) q(x) \mathrm{d} x+\frac{1}{2} \int_{\mathbb{R}^{n}} \int_{\mathbb{R}^{n}} V(q, t)(\xi, \eta) q(\xi) q(\eta) h(\xi) \cdot h(\eta) \mathrm{d} \xi \mathrm{d} \eta \\
\quad+\int_{\mathbb{R}^{n}} q(x)\left(f(x, \hat{v}(q, U))+D_{x} U(x, q) \cdot g(x, \hat{v}(q, U))\right) \mathrm{d} x=0, \\
\Phi(q, T)=\int_{\mathbb{R}^{n}} f_{T}(x) q(x) \mathrm{d} x .
\end{array}\right.
$$

The Master equation is an equation for $U(x, q, t)$. It is obtained by differentiating (3.16) with respect to $q$. We therefore obtain, formally:

$$
\left\{\begin{array}{l}
\frac{\partial U}{\partial t}-A_{x} U-\int_{\mathbb{R}^{n}} A_{\xi} V(q, t)(x, \xi) q(\xi) \mathrm{d} \xi+h(x) \cdot \int_{\mathbb{R}^{n}} V(q, t)(x, \xi) h(\xi) q(\xi) \mathrm{d} \xi \\
\quad+\frac{1}{2} \int_{\mathbb{R}^{n}} \int_{\mathbb{R}^{n}} \frac{\partial V}{\partial q}(q, t)(\xi, \eta)(x) h(\xi) \cdot h(\eta) q(\xi) q(\eta) \mathrm{d} \xi \mathrm{d} \eta+f(x, \hat{v}(q, U)) \\
\quad+D_{x} U \cdot g(x, \hat{v}(q, U))+\int_{\mathbb{R}^{n}} D_{\xi} V(q, t)(\xi, x) \cdot g(\xi, \hat{v}(q, U)) q(\xi) \mathrm{d} \xi=0, \\
U(x, q, T)=f_{T}(x) .
\end{array}\right.
$$

Note that

$$
\frac{\partial V}{\partial q}(q, t)(\xi, \eta)(x)=\frac{\partial^{3} \Phi}{\partial q^{3}}(q, t)(x, \xi, \eta),
$$

which is symmetric in the arguments $(x, \xi, \eta)$. 


\subsection{System of HJB-FP equations}

In Mean Field Theory approach, the Master equation is the key equation. However, it is an infinite-dimensional nonlinear PDE. Direct approaches are very limited. The most convenient approach is to use ideas similar to the classical method of characteristics. This amounts to solving a system of forward-backward finite dimensional stochastic PDE. Since it is forward-backward the initial conditions matter. We shall consider that the initial time is 0 , for convenience; yet the same argument applies for any time $t \in[0, T]$. This system is called HamiltonJacobi-Bellman for the backward equation and Fokker-Planck for the forward one. The Fokker-Planck equation is the Zakai equation in which we insert the optimal feedabck $\hat{v}(q, U)$. So we get

$$
\left\{\begin{array}{l}
\mathrm{d} q+A^{*} q(x, t) \mathrm{d} t+\operatorname{div}(g(x, \hat{v}(q, U)) q(x, t)) \mathrm{d} t-q(x, t) h(x) \cdot \mathrm{d} z(t)=0, \\
q(x, 0)=q(x)
\end{array}\right.
$$

The functional $U(x, q, t)$ used in (3.19) is the functional solution of the master equation (3.17). We call simply $q(t)$ the solution of (3.19). We then set

$$
u(x, t)=U(x, q(t), t) .
$$

We use the notation $\hat{v}\left(q_{t}, u_{t}\right)$ to represent the functional $\hat{v}(q, U)$ in which the arguments $q, U$ are replaced by $q(\cdot, t)$ and $U(\cdot, q(\cdot, t), t)=u(\cdot, t)$. We denote $q_{t}=q(\cdot, t), u_{t}=u(\cdot, t)$ to simplify. The functional $\hat{v}\left(q_{t}, u_{t}\right)$ achieves the infimum of

$$
\hat{v}\left(q_{t}, u_{t}\right)=\arg \min _{v} \int_{\mathbb{R}^{n}} q(x, t)\left(f(x, v)+D_{x} u(x, t) \cdot g(x, v)\right) \mathrm{d} x .
$$

The next step is to obtain the equation for $u(x, t)$. It is a long and tedious calculation, obtained in taking the Ito differential of the random field defined by (3.20). We omit the detail and only give the result as follows:

$$
\left\{\begin{array}{l}
-d u+\left(A u-D u \cdot g\left(x, \hat{v}\left(q_{t}, u_{t}\right)\right)\right) \mathrm{d} t=f\left(x, \hat{v}\left(q_{t}, u_{t}\right)\right)-K(x, t) \cdot(\mathrm{d} z(t)-h(x) \mathrm{d} t), \\
u(x, T)=f_{T}(x),
\end{array}\right.
$$

where $K(x, t)$ is defined by the formula:

$$
K(x, t)=\int_{\mathbb{R}^{n}} V\left(q_{t}, t\right)(x, \xi) h(\xi) q(\xi, t) \mathrm{d} \xi
$$

In fact, we do not need to compute $K(x, t)$ by formula (3.23), which would require the knowledge of $V\left(q_{t}, t\right)(x, \xi)$, thus solving the master equation. From the theory of backward stochastic PDE the random field $K(x, t)$ is required by the condition of adaptativity of $u(x, t)$. So the solution of $(3.22)$ is not just $u(x, t)$ but the pair $(u(x, t), K(x, t))$, and we can expect uniqueness. Equation (3.22) is the HJB equation. It must be coupled with the FP equation (3.19) written as follows:

$$
\left\{\begin{array}{l}
\mathrm{d} q+A^{*} q(x, t) \mathrm{d} t+\operatorname{div}\left(g\left(x, \hat{v}\left(q_{t}, u_{t}\right)\right) q(x, t)\right) \mathrm{d} t-q(x, t) h(x) \cdot \mathrm{d} z(t)=0, \\
q(x, 0)=q(x)
\end{array}\right.
$$

recalling also (3.21). So the pair (3.22), (3.24) is the pair of HJB-FP equations. Since $q(x, 0)=q(x)$, we can assert that

$$
u(x, 0)=U(x, q, 0) .
$$


Therefore we can compute $U(x, q, 0)$ by solving the system of HJB-FP equations and using formula (3.25). Of course $u(x, t) \neq U(x, q, t)$. To compute $U(x, q, t)$, we have to write the system (3.22), (3.24) on the interval $(t, T)$ instead of $(0, T)$. In that sense, the system of HJB-FP equations (3.22), (3.24) is a method of characteristics to solve the master equation (3.17). Besides the optimal optimal feedback $\hat{v}(q, U(\cdot, q, t), t)$ can be derived from the system of HJB-FP equations; indeed,

$$
\hat{v}(q, U(\cdot, q, 0), 0)=\hat{v}(q, u(\cdot, 0)),
$$

and setting the initial condition of the system of HJB-FP equations at $t$ instead of 0 yields $\hat{v}(q, U(\cdot, q, t), t)$. To compute the value function, we have to rely on Bellman equation (3.16). Let us compute $\frac{\partial \Phi}{\partial t}(q, 0)$, by using (3.16). The only term which is not known is $\int_{\mathbb{R}^{n}} \int_{\mathbb{R}^{n}} V(q, 0)(\xi, \eta) q(\xi) q(\eta) h(\xi) \cdot h(\eta) \mathrm{d} \xi \mathrm{d} \eta$. However from (3.23), we can write

$$
\int_{\mathbb{R}^{n}} \int_{\mathbb{R}^{n}} V(q, 0)(\xi, \eta) q(\xi) q(\eta) h(\xi) \cdot h(\eta) \mathrm{d} \xi \mathrm{d} \eta=\int_{\mathbb{R}^{n}} h(\xi) \cdot K(\xi, 0) q(\xi) \mathrm{d} \xi .
$$

Collecting results, we can write the formula:

$$
\begin{aligned}
\frac{\partial \Phi}{\partial t}(q, 0)= & \int_{\mathbb{R}^{n}} A_{x} u(x, 0) q(x) \mathrm{d} x-\frac{1}{2} \int_{\mathbb{R}^{n}} h(x) \cdot K(x, 0) q(x) \mathrm{d} x \\
& -\int_{\mathbb{R}^{n}} q(x)\left(f(x, \hat{v}(q, u(\cdot, 0)))+D_{x} u(x, 0) \cdot g(x, \hat{v}(q, u(\cdot, 0)))\right) \mathrm{d} x .
\end{aligned}
$$

In a similar way, we can define $\frac{\partial \Phi}{\partial t}(q, t)$, for any $t$ and any $q$. Since we know $\Phi(q, T)$, we obtain $\Phi(q, t)$ for any $t$. So solving the system of HJB-FP equations provides all the information on the value function and on the optimal feedback.

\section{WEAK FORMULATION OF ZAKAi EQUATION}

\subsection{Weak formulation and linear dynamics}

In this section, we consider Zakai equation as follows:

$$
\left\{\begin{array}{l}
\mathrm{d} q+A^{*} q(x, t) \mathrm{d} t+\operatorname{div}(g(x, v(t)) q(x, t)) \mathrm{d} t-q(x, t) h(x) \cdot \mathrm{d} z(t)=0, \\
q(x, 0)=q(x)
\end{array}\right.
$$

in which $v(t)$ is a fixed process adapted to the filtration $\mathcal{Z}^{t}=\sigma(z(s), 0 \leq s \leq t)$. If $\psi(x, t)$ is a deterministic function in $(x, t)$ which is $C^{2}$ in $x$ and $C^{1}$ in $t$, we deduce immediately from (4.1), by simple integration-by-parts that

$$
\int_{\mathbb{R}^{n}} \psi(x, t) \mathrm{d} q=\int_{\mathbb{R}^{n}} q(x, t)[-A \psi(x, t)+g(x, v(t)) \cdot D \psi(x, t)] \mathrm{d} t+\int_{\mathbb{R}^{n}} q(x, t) \psi(x, t) h(x) \cdot \mathrm{d} z(t),
$$

and thus

$$
\begin{aligned}
\int_{\mathbb{R}^{n}} q(x, t) \psi(x, t) \mathrm{d} x= & \int_{\mathbb{R}^{n}} q(x) \psi(x, 0) \mathrm{d} x+\int_{0}^{t} \int_{\mathbb{R}^{n}} q(x, s)\left(\frac{\partial \psi}{\partial s}-A \psi(x, s)+g(x, v(s)) \cdot D \psi(x, s)\right) \mathrm{d} x \mathrm{~d} s \\
& +\int_{0}^{t} \int_{\mathbb{R}^{n}} q(x, s) \psi(x, s) h(x) \cdot \mathrm{d} z(s),
\end{aligned}
$$


which is the weak formulation of Zakai equation. Note that the formulation (4.1) (strong form) and the weak form (4.2) are not equivalent. We may have a weak solution but not a strong solution.

\subsection{Linear system and linear observation}

We want to solve Zakai equation in the following special case:

$$
\left\{\begin{array}{l}
g(x, v)=F x+G v, \sigma(x)=\sigma \\
h(x)=H x
\end{array}\right.
$$

In general, this case is associated to an initial probability $q(x)$, which is Gaussian. In our approach, we cannot take a special $q(x)$. It must remain general, because it is an argument of the value function and of the solution of the master equation. When we solve the system of HJB-FP equations, we can take $q(x)$ Gaussian, but then we cannot use this method to obtain the solution of the master equation or of Bellman equation. For a given control $v(t)$ which is a process adapted to $\mathcal{Z}^{t}$, Zakai equation reads:

$$
\left\{\begin{array}{l}
\left.\mathrm{d} q-\operatorname{tr}\left(a D_{x}^{2} q(x, t)\right) \mathrm{d} t+\operatorname{div}(F x+G v(t)) q(x, t)\right) \mathrm{d} t-q(x, t) H x \cdot \mathrm{d} z(t)=0 \\
q(x, 0)=q(x)
\end{array}\right.
$$

where $a=\frac{1}{2} \sigma \sigma^{*}$. Makowski [12] has shown that this equation has an explicit solution, that we describe now, in a weak form. We first need some notations. We introduce the matrix $\Sigma(t)$ solution of the Riccati equation:

$$
\left\{\begin{array}{l}
\frac{\mathrm{d} \Sigma}{\mathrm{d} t}+\Sigma(t) H^{*} H \Sigma(t)-F \Sigma(t)-\Sigma(t) F^{*}=2 a \\
\Sigma(0)=0
\end{array}\right.
$$

We then define the matrix solution $\Phi(t)$ of the differential equation:

$$
\left\{\begin{array}{l}
\frac{\mathrm{d} \Phi}{\mathrm{d} t}=\left(F-\Sigma(t) H^{*} H\right) \Phi(t) \\
\Phi(0)=I
\end{array}\right.
$$

and

$$
S(t)=\int_{0}^{t} \Phi^{*}(s) H^{*} H \Phi(s) \mathrm{d} s
$$

We then introuduce stochastic processes $\beta(t)$ and $\rho(t)$ adapted to the filtration $\mathcal{Z}^{t}$, defined by the following respective equations

$$
\begin{gathered}
\left\{\begin{array}{l}
\mathrm{d} \beta(t)= \\
\beta(0)=
\end{array}\right. \\
\qquad\left\{\begin{array}{l}
\mathrm{d} \rho(t)=\Phi^{*}(t) H^{*}(\mathrm{~d} z(t)-H \beta(t)) \mathrm{d} t+\Sigma(t) H^{*}(\mathrm{~d} z-H \beta(t) \mathrm{d} t) \\
\rho(0)=0 .
\end{array}\right.
\end{gathered}
$$


The process $\beta(t)$ is the Kalman filter for the linear system (4.3) with a deterministic initial condition, equal to 0 . If we set

$$
m(x, t)=\Phi(t) x+\beta(t)
$$

we obtain the Kalman filter for the same linear dynamic system, with an intial condition $x$. It satisfies the equation:

$$
\left\{\begin{array}{l}
d_{t} m(x, t)=(F m(x, t)+G v(t)) \mathrm{d} t+\Sigma(t) H^{*}(\mathrm{~d} z-H m(x, t) \mathrm{d} t) \\
m(x, 0)=x
\end{array}\right.
$$

Finally, we introduce the martingale $\theta(x, t)$ defined by:

$$
\left\{\begin{array}{l}
d_{t} \theta(x, t)=\theta(x, t) H m(x, t) \cdot \mathrm{d} z(t), \\
\theta(x, 0)=1
\end{array}\right.
$$

whose solution is the Doléans-Dade exponential

$$
\theta(x, t)=\exp \left(\int_{0}^{t} H m(x, s) \cdot \mathrm{d} z(s)-\frac{1}{2} \int_{0}^{t}|H m(x, s)|^{2} \mathrm{~d} s\right) .
$$

\subsection{Formulas}

We can state the following result, due to Makowski [12], whose proof can be found in [3].

Proposition 4.1. For any test function $\psi(x, t)$, we have

$$
\int_{\mathbb{R}^{n}} q(x, t) \psi(x, t) \mathrm{d} x=\int_{\mathbb{R}^{n}} \theta(x, t)\left(\int_{\mathbb{R}^{n}} \psi\left(m(x, t)+\Sigma(t)^{\frac{1}{2}} \xi, t\right) \frac{\exp \left(-\frac{|\xi|^{2}}{2}\right)}{(2 \pi)^{\frac{n}{2}}} \mathrm{~d} \xi\right) q(x) \mathrm{d} x .
$$

Proof. Equality (4.14) is true for $t=0$. Let us set

$$
\mathcal{L} \psi(x, t)=\frac{\partial \psi}{\partial s}-A \psi(x, t)+g(x, v(t)) \cdot D \psi(x, t)
$$

According to (4.2), it is thus sufficient to show that

$$
\begin{aligned}
& d_{t} \theta(x, t)\left(\int_{\mathbb{R}^{n}} \psi\left(m(x, t)+\Sigma(t)^{\frac{1}{2}} \xi, t\right) \exp -\frac{|\xi|^{2}}{2} \mathrm{~d} \xi\right) \\
= & \theta(x, t) \int_{\mathbb{R}^{n}} \mathcal{L} \psi\left(m(x, t)+\Sigma(t)^{\frac{1}{2}} \xi, t\right) \exp \left(-\frac{|\xi|^{2}}{2}\right) \mathrm{d} \xi \mathrm{d} t+\theta(x, t) \int_{\mathbb{R}^{n}} \psi\left(m(x, t)+\Sigma(t)^{\frac{1}{2}} \xi, t\right) H\left(m(x, t)+\Sigma(t)^{\frac{1}{2}} \xi\right) \exp -\frac{|\xi|^{2}}{2} \mathrm{~d} \xi \cdot \mathrm{d} z(t) .
\end{aligned}
$$

This can be done through some tedious calculation, whose details can be found in [3].

We shall derive from (4.14) a more analytic formula. We first set

$$
\nu(t)=\int_{\mathbb{R}^{n}} q(x, t) \mathrm{d} x=\int_{\mathbb{R}^{n}} \theta(x, t) q(x) \mathrm{d} x .
$$


Hence, from (4.12),

$$
\mathrm{d} \nu(t)=\int_{\mathbb{R}^{n}} \theta(x, t) H m(x, t) q(x) \mathrm{d} x \cdot \mathrm{d} z(t) .
$$

But from (4.14), we see that

$$
\int_{\mathbb{R}^{n}} q(x, t) x \mathrm{~d} x=\int_{\mathbb{R}^{n}} \theta(x, t) m(x, t) q(x) \mathrm{d} x .
$$

Therefore,

$$
\mathrm{d} \nu(t)=H \int_{\mathbb{R}^{n}} q(x, t) x \mathrm{~d} x \cdot \mathrm{d} z(t)=\nu(t) H \hat{x}(t) \cdot \mathrm{d} z(t)
$$

where we have set

$$
\hat{x}(t)=\frac{\int_{\mathbb{R}^{n}} q(x, t) x \mathrm{~d} x}{\int_{\mathbb{R}^{n}} q(x, t) \mathrm{d} x} .
$$

Referring to (2.8), we see that

$$
\hat{x}(t)=\mathbb{E}^{v(\cdot)}\left[x(t) \mid \mathcal{Z}^{t}\right]
$$

the conditional mean of the process $x(t)$ defined by, see (2.1):

$$
\left\{\begin{array}{l}
\mathrm{d} x=(F x(t)+G v(t)) \mathrm{d} t+\sigma \mathrm{d} w, \\
x(0)=\xi,
\end{array}\right.
$$

with respect to the filtration $\mathcal{Z}^{t}$ on the probability space $\left(\Omega, \mathcal{A}, \mathbb{P}^{v(\cdot)}\right)$. It is thus the Kalman filter in this probabilistic set up. We shall derive the form of its evolution in the sequel. Now, from (4.17) we can assert that:

$$
\nu(t)=\exp \left\{\int_{0}^{t} H \hat{x}(s) \cdot \mathrm{d} z(s)-\frac{1}{2} \int_{0}^{t}|H \hat{x}(s)|^{2} \mathrm{~d} s\right\} \int_{\mathbb{R}^{n}} q(x) \mathrm{d} x
$$

recalling that, see (4.15), $\nu(0)=\int_{\mathbb{R}^{n}} q(x) \mathrm{d} x$. Next, from (4.13) and (4.10), we have:

$$
\begin{aligned}
\theta(x, t) & =\exp \left(\int_{0}^{t} H(\Phi(s) x+\beta(s)) \cdot \mathrm{d} z(s)-\frac{1}{2} \int_{0}^{t}|H(\Phi(s) x+\beta(s))|^{2} \mathrm{~d} s\right) \\
& =\gamma(t) \exp \left(-\frac{x^{*} S(t) x-2 x^{*} \rho(t)}{2}\right),
\end{aligned}
$$

with

$$
\gamma(t)=\exp \left\{\int_{0}^{t} H \beta(s) \cdot \mathrm{d} z(s)-\frac{1}{2} \int_{0}^{t}|H \beta(s)|^{2} \mathrm{~d} s\right\}
$$


and recalling the definition of $S(t)$ and $\rho(t)$, see (4.7) and (4.9). From (4.15), we obtain:

$$
\nu(t)=\gamma(t) \int_{\mathbb{R}^{n}} \exp \left(-\frac{x^{*} S(t) x-2 x^{*} \rho(t)}{2}\right) q(x) \mathrm{d} x .
$$

Combining results, we can assert that:

$$
\theta(x, t)=\nu(t) \frac{\exp \left(-\frac{x^{*} S(t) x-2 x^{*} \rho(t)}{2}\right)}{\int_{\mathbb{R}^{n}} \exp \left(-\frac{\xi^{*} S(t) \xi-2 \xi^{*} \rho(t)}{2}\right) q(\xi) \mathrm{d} \xi} .
$$

Next, using (4.16) and (4.10), we have:

$$
\begin{aligned}
\int_{\mathbb{R}^{n}} q(x, t) x \mathrm{~d} x & =\int_{\mathbb{R}^{n}} \theta(x, t)(\Phi(t) x+\beta(t)) q(x) \mathrm{d} x \\
& =\Phi(t) \int_{\mathbb{R}^{n}} \theta(x, t) x q(x) \mathrm{d} x+\beta(t) \int_{\mathbb{R}^{n}} \theta(x, t) q(x) \mathrm{d} x,
\end{aligned}
$$

therefore, from (4.18) we also obtain:

$$
\hat{x}(t)=\Phi(t) \frac{\int_{\mathbb{R}^{n}} \theta(x, t) x q(x) \mathrm{d} x}{\int_{\mathbb{R}^{n}} \theta(x, t) q(x) \mathrm{d} x}+\beta(t) .
$$

Let us introduce the deterministic function of arguments $\rho \in \mathbb{R}^{n}$ and $t$ :

$$
b(\rho, t)=\frac{\int_{\mathbb{R}^{n}} x \exp \left(-\frac{x^{*} S(t) x-2 x^{*} \rho}{2}\right) q(x) \mathrm{d} x}{\int_{\mathbb{R}^{n}} \exp \left(-\frac{x^{*} S(t) x-2 x^{*} \rho}{2}\right) q(x) \mathrm{d} x},
$$

then (4.22) can be written as:

$$
\hat{x}(t)=\Phi(t) b(\rho(t), t)+\beta(t),
$$

We can finally state the main formula for the unnormalized conditional probability $q(x, t)$.

Theorem 4.2. The unnormalized conditional probability $q(x, t)$ is given by:

$$
\begin{aligned}
\int_{\mathbb{R}^{n}} q(x, t) \psi(x, t) \mathrm{d} x= & \frac{\nu(t)}{\int_{\mathbb{R}^{n}} \exp \left(-\frac{x^{*} S(t) x-2 x^{*} \rho(t)}{2}\right) q(x) \mathrm{d} x} \int_{\mathbb{R}^{n}} \exp \left(-\frac{x^{*} S(t) x-2 x^{*} \rho(t)}{2}\right) \\
& \cdot\left[\int_{\mathbb{R}^{n}} \psi\left(\hat{x}(t)+\Phi(t)(x-b(\rho(t), t))+\Sigma(t)^{\frac{1}{2}} \xi, t\right) \frac{\exp \left(-\frac{|\xi|^{2}}{2}\right)}{(2 \pi)^{\frac{n}{2}}} \mathrm{~d} \xi\right] q(x) \mathrm{d} x .
\end{aligned}
$$

\subsection{Sufficient statistics}

We see, from formula (4.25), that the unnormalized conditional probability $q(x, t)$ is completely characterized by two processes $\hat{x}(t)$ and $\rho(t)$, which are stochastic processes adapted to $\mathcal{Z}^{t}$ with values in $\mathbb{R}^{n}$, and it is crucial 
to obtain their evolutions. To this end, we need to introduce a new function $B(\rho, t)$ similar to $b(\rho, t)$ defined by the following formula:

$$
B(\rho, t):=\frac{\int_{\mathbb{R}^{n}} x x^{*} \exp \left(-\frac{x^{*} S(t) x-2 x^{*} \rho}{2}\right) q(x) \mathrm{d} x}{\int_{\mathbb{R}^{n}} \exp \left(-\frac{\xi^{*} S(t) \xi-2 \xi^{*} \rho(t)}{2}\right) q(\xi) \mathrm{d} \xi},
$$

and we also define

$$
\Gamma(\rho, t):=\Sigma(t)+\Phi(t)\left(B(\rho, t)-b(\rho, t) b^{*}(\rho, t)\right) \Phi^{*}(t) .
$$

We are going to show that:

Proposition 4.3. The pair $(\hat{x}(t), \rho(t))$ is the solution of the following systems of SDEs:

$$
\begin{gathered}
\left\{\begin{array}{l}
\mathrm{d} \hat{x}(t)=(F \hat{x}(t)+G v(t)) \mathrm{d} t+\Gamma(\rho(t), t) H^{*}(\mathrm{~d} z(t)-H \hat{x}(t) \mathrm{d} t), \\
\hat{x}(0)=\frac{\int_{\mathbb{R}^{n}} x q(x) \mathrm{d} x}{\int_{\mathbb{R}^{n}} q(x) \mathrm{d} x} ;
\end{array}\right. \\
\left\{\begin{array}{l}
\mathrm{d} \rho(t)=\Phi^{*}(t) H^{*}(\mathrm{~d} z(t)-H(\hat{x}(t)-\Phi(t) b(\rho(t), t))), \\
\rho(0)=0 .
\end{array}\right.
\end{gathered}
$$

Proof. The pair $(\rho(t), \beta(t))$ satisfies (4.8), (4.9) and $\hat{x}(t)$ satisfies (4.24), therefore

$$
\mathrm{d} \hat{x}(t)=\mathrm{d} \beta(t)+\frac{\mathrm{d} \Phi(t)}{\mathrm{d} t} b(\rho(t), t) \mathrm{d} t+\Phi(t) \mathrm{d} b(\rho(t), t) .
$$

Next, we use

$$
\begin{gathered}
D_{\rho} b(\rho, t)=B(\rho, t)-b(\rho, t) b^{*}(\rho, t), \\
\operatorname{tr}\left(D_{\rho}^{2} b(\rho, t) L\right)=-B(\rho, t)\left(L+L^{*}\right) b(\rho, t)-\operatorname{tr}(B(\rho, t) L) b(\rho, t)+2 b(\rho, t) b^{*}(\rho, t) L b(\rho, t) \\
+\frac{\int_{\mathbb{R}^{n}} x\left(x^{*} L x\right) \exp \left(-\frac{x^{*} S(t) x-2 x^{*} \rho}{2}\right) q(x) \mathrm{d} x}{\int_{\mathbb{R}^{n}} \exp \left(-\frac{x^{*} S(t) x-2 x^{*} \rho}{2}\right) q(x) \mathrm{d} x},
\end{gathered}
$$

for any dummy matrix $L$. Therefore,

$$
\begin{aligned}
\frac{1}{2} \operatorname{tr}\left(D_{\rho}^{2} b(\rho, t) \Phi^{*}(t) H^{*} H \Phi(t)\right)= & -B(\rho, t) \Phi^{*}(t) H^{*} H \Phi(t) b(\rho, t)-\frac{1}{2} \operatorname{tr}\left(B(\rho, t) \Phi^{*}(t) H^{*} H \Phi(t)\right) b(\rho, t) \\
& +\operatorname{tr}\left(b(\rho, t) b^{*}(\rho, t) \Phi^{*}(t) H^{*} H \Phi(t)\right) b(\rho, t) \\
& +\frac{1}{2} \frac{\int_{\mathbb{R}^{n}} x\left(x^{*} \Phi^{*}(t) H^{*} H \Phi(t) x\right) \exp \left(-\frac{x^{*} S(t) x-2 x^{*} \rho}{2}\right) q(x) \mathrm{d} x}{\int_{\mathbb{R}^{n}} \exp \left(-\frac{x^{*} S(t) x-2 x^{*} \rho}{2}\right) q(x) \mathrm{d} x} .
\end{aligned}
$$


Also,

$$
\frac{\partial b(\rho, t)}{\partial t}=\frac{1}{2} \operatorname{tr}\left(B(\rho, t) \Phi^{*}(t) H^{*} H \Phi(t)\right) b(\rho, t)-\frac{1}{2} \frac{\int_{\mathbb{R}^{n}} x\left(x^{*} \Phi^{*}(t) H^{*} H \Phi(t) x\right) \exp \left(-\frac{x^{*} S(t) x-2 x^{*} \rho}{2}\right) q(x) \mathrm{d} x}{\int_{\mathbb{R}^{n}} \exp \left(-\frac{x^{*} S(t) x-2 x^{*} \rho}{2}\right) q(x) \mathrm{d} x} .
$$

Hence,

$\frac{\partial b(\rho, t)}{\partial t}+\frac{1}{2} \operatorname{tr}\left(D_{\rho}^{2} b(\rho, t) \Phi^{*}(t) H^{*} H \Phi(t)\right)=-B(\rho, t) \Phi^{*}(t) H^{*} H \Phi(t) b(\rho, t)+\operatorname{tr}\left(b(\rho, t) b^{*}(\rho, t) \Phi^{*}(t) H^{*} H \Phi(t)\right) b(\rho, t)$.

We can then compute $\mathrm{d} b(\rho(t), t)$ by making use of $(4.34),(4.31)$ and $(4.29)$, we then obtain:

$$
\mathrm{d} b(\rho(t), t)=\left(B(\rho(t), t)-b(\rho(t), t) b^{*}(\rho(t), t)\right) \Phi^{*}(t) H^{*}(\mathrm{~d} z(t)-H \hat{x}(t) \mathrm{d} t) .
$$

Using (4.6), (4.30) and (4.35), we obtain easily (4.28), recalling the definition of $\Gamma(\rho, t)$, see (4.27). The relation (4.29) follows immediately from (4.9) and (4.24). The proof is complete.

We also have the follwing interpretation of $\Gamma(\rho(t), t)$ as the conditional variance of the process $x(t)$ :

Proposition 4.4. We have the formula:

$$
\Gamma(\rho(t), t)=\frac{\int_{\mathbb{R}^{n}} x x^{*} q(x, t) \mathrm{d} x}{\int_{\mathbb{R}^{n}} q(x, t) \mathrm{d} x}-\hat{x}(t) \hat{x}(t)^{*}
$$

Proof. We use (4.25) to write:

$$
\begin{aligned}
& \frac{\int_{\mathbb{R}^{n}} x x^{*} q(x, t) \mathrm{d} x}{\int_{\mathbb{R}^{n}} q(x, t) \mathrm{d} x} \\
= & \frac{1}{\int_{\mathbb{R}^{n}} \exp \left(-\frac{x^{*} S(t) x-2 x^{*} \rho(t)}{2}\right) q(x) \mathrm{d} x} \int_{\mathbb{R}^{n}} \exp \left(-\frac{x^{*} S(t) x-2 x^{*} \rho(t)}{2}\right) \\
& \cdot\left[\int_{\mathbb{R}^{n}}\left(\hat{x}(t)+\Phi(t)(x-b(\rho(t), t))+\Sigma(t)^{\frac{1}{2}} \xi\right)\left(\hat{x}(t)+\Phi(t)(x-b(\rho(t), t))+\Sigma(t)^{\frac{1}{2}} \xi\right)^{*} \frac{\exp \left(-\frac{|\xi|^{2}}{2}\right)}{(2 \pi)^{\frac{n}{2}}} \mathrm{~d} \xi\right] q(x) \mathrm{d} x \\
= & \frac{1}{\int_{\mathbb{R}^{n}} \exp \left(-\frac{x^{*} S(t) x-2 x^{*} \rho(t)}{2}\right) q(x) \mathrm{d} x} \\
& \cdot \int_{\mathbb{R}^{n}} \exp \left(-\frac{x^{*} S(t) x-2 x^{*} \rho(t)}{2}\right) \cdot\left[((\hat{x}(t)+\Phi(t)(x-b(\rho(t), t))))((\hat{x}(t)+\Phi(t)(x-b(\rho(t), t))))^{*}+\Sigma(t)\right] \\
= & \hat{x}(t) \hat{x}(t)^{*}+\Phi(t)(B(\rho(t), t)-b(\rho(t), t))(B(\rho(t), t)-b(\rho(t), t))^{*} \Phi(t)^{*}+\Sigma(t) \\
= & \hat{x}(t) \hat{x}(t)^{*}+\Gamma(\rho(t), t),
\end{aligned}
$$

which is (4.36) as desired. 


\subsection{The Gaussian case}

We first begin by giving the characteristic function (Fourier transform) of the unnormalized probability density denoted by:

$$
\begin{aligned}
\hat{Q}(\lambda, t) & =\int_{\mathbb{R}^{n}} q(x, t) \exp \left(i \lambda^{*} x\right) \mathrm{d} x \\
& =\nu(t) \exp \left[-\frac{1}{2} \lambda^{*} \Sigma(t) \lambda+i \lambda^{*}(\hat{x}(t)-\Phi(t) b(\rho(t), t))\right] \cdot \frac{\int_{\mathbb{R}^{n}} \exp \left(-\frac{x^{*} S(t) x-2 x^{*}\left(\rho(t)+i \Phi^{*}(t) \lambda\right)}{2}\right) q(x) \mathrm{d} x}{\int_{\mathbb{R}^{n}} \exp \left(-\frac{x^{*} S(t) x-2 x^{*} \rho(t)}{2}\right) q(x) \mathrm{d} x} .
\end{aligned}
$$

The Gaussian case corresponds to an initial value of the system (4.19) which is Gaussian:

$$
q(x)=\frac{\exp \left(-\frac{1}{2}\left(x-\bar{x}_{0}\right)^{*} P_{0}^{-1}\left(x-\bar{x}_{0}\right)\right)}{(2 \pi)^{\frac{n}{2}}\left|P_{0}\right|^{\frac{1}{2}}}
$$

where we have assumed the initial variance $P_{0}$ to be invertible, to simplify calculations. Using (4.23), we obtain:

$$
\begin{aligned}
b(\rho, t) & =\left(S(t)+P_{0}^{-1}\right)^{-1}\left(\rho+P_{0}^{-1} \bar{x}_{0}\right), \\
B(\rho, t) & =b(\rho, t) b(\rho, t)^{*}+\left(S(t)+P_{0}^{-1}\right)^{-1} .
\end{aligned}
$$

Therefore, from (4.27), we obtain:

$$
\Gamma(\rho, t)=\Sigma(t)+\Phi(t)\left(S(t)+P_{0}^{-1}\right)^{-1} \Phi(t)^{*}=P(t)
$$

which is independent of $\rho$. An easy calculation shows that $P(t)$ is the solution of the Riccati equation:

$$
\left\{\begin{array}{l}
\frac{\mathrm{d} P}{\mathrm{~d} t}+P H^{*} H P-F P-P F^{*}=2 a \\
P(0)=P_{0}
\end{array}\right.
$$

and $\hat{x}(t)$ is then the classical Kalman filter:

$$
\left\{\begin{array}{l}
\mathrm{d} \hat{x}(t)=(F \hat{x}(t)+G v(t)) \mathrm{d} t+P(t) H^{*}(\mathrm{~d} z(t)-H \hat{x}(t) \mathrm{d} t) \\
\hat{x}(0)=\bar{x}_{0}
\end{array}\right.
$$

To obtain $q(x, t)$, we use the characteristic function (4.37). An easy calculation yields:

$$
\hat{Q}(\lambda, t)=\nu(t) \exp \left(i \lambda^{*} \hat{x}(t)-\frac{1}{2} \lambda^{*} P(t) \lambda\right)
$$

which is the characteristic function of a Gaussian random variable with mean $\hat{x}(t)$ and variance $P(t)$. Recall that it is a conditional probability density given $\mathcal{Z}^{t}$. 


\section{LINEAR QUADRATIC CONTROL PROBLEM}

\subsection{Setting of the problem}

We want to apply the theory developed in Section 3 to the linear dynamics and linear observation (4.3), with a quadratic cost:

$$
\left\{\begin{array}{l}
f(x, v)=x^{*} M x+v^{*} N v \\
f_{T}(x)=x^{*} M_{T} x
\end{array}\right.
$$

in which $M$ and $M_{T}$ are $n \times n$ symmetric positive semi-definite matrices and $N$ is an $m \times m$ symmetric positive definte matrix. We want to solve the control problem (2.9), (2.10) in this case. We write it as follows:

$$
\begin{gathered}
\left\{\begin{array}{l}
\left.\mathrm{d} q-\operatorname{tr}\left(a D_{x}^{2} q(x, t)\right) \mathrm{d} t+\operatorname{div}(F x+G v(t)) q(x, t)\right) \mathrm{d} t-q(x, t) H x \cdot \mathrm{d} z(t)=0, \\
q(x, 0)=q(x),
\end{array}\right. \\
J(v(\cdot))=\mathbb{E}\left[\int_{0}^{T} \int_{\mathbb{R}^{n}} q^{v(\cdot)}(x, t)\left(x^{*} M x+v(t)^{*} N v(t)\right) \mathrm{d} x \mathrm{~d} t+\int_{\mathbb{R}^{n}} q^{v(\cdot)}(x, T) x^{*} M_{T} x \mathrm{~d} x\right] .
\end{gathered}
$$

In the sequel, we shall drop the index $v(\cdot)$ in $q(x, t)$ without cause of too much ambiguity.

\subsection{Application of mean field theory}

We begin by finding the function $\hat{v}(q, U)$ defined by (3.13). We have to solve for the minimization problem:

$$
\inf _{v}\left[v^{*} N v \int_{\mathbb{R}^{n}} q(x) \mathrm{d} x+G v \cdot \int_{\mathbb{R}^{n}} D_{x} U(x, q) q(x) \mathrm{d} x\right]
$$

which yields

$$
\hat{v}(q, U)=-\frac{1}{2} N^{-1} G^{*} \frac{\int_{\mathbb{R}^{n}} D_{x} U(x, q) q(x) \mathrm{d} x}{\int_{\mathbb{R}^{n}} q(x) \mathrm{d} x},
$$

and thus

$\inf _{v}\left[v^{*} N v \int_{\mathbb{R}^{n}} q(x) \mathrm{d} x+G v \cdot \int_{\mathbb{R}^{n}} D_{x} U(x, q) q(x) \mathrm{d} x\right]=-\frac{1}{4} \frac{\int_{\mathbb{R}^{n}} D_{x} U(x, q) q(x) \mathrm{d} x \cdot G N^{-1} G^{*} \int_{\mathbb{R}^{n}} D_{x} U(x, q) q(x) \mathrm{d} x}{\int_{\mathbb{R}^{n}} q(x) \mathrm{d} x}$.

We consider the value function:

$$
\Phi(q, t)=\inf _{v(\cdot)} J(v(\cdot))
$$


and we write Bellman equation (3.16) specifically for the linear quadratic case as follows:

$$
\left\{\begin{array}{l}
\frac{\partial \Phi}{\partial t}+\operatorname{tr}\left(a \int_{\mathbb{R}^{n}} D_{x}^{2} U(x, q, t) q(x)\right) \mathrm{d} x+\frac{1}{2} \int_{\mathbb{R}^{n}} \int_{\mathbb{R}^{n}} V(q, t)(\xi, \eta) H \xi \cdot H \eta q(\xi) q(\eta) \mathrm{d} \xi \mathrm{d} \eta+\int_{\mathbb{R}^{n}} x^{*} M x q(x) \mathrm{d} x \\
\quad+\int_{\mathbb{R}^{n}} F x \cdot D_{x} U(x, q, t) q(x) \mathrm{d} x-\frac{1}{4} \frac{\int_{\mathbb{R}^{n}} D_{x} U(x, q, t) q(x) \mathrm{d} x \cdot G N^{-1} G^{*} \int_{\mathbb{R}^{n}} D_{x} U(x, q, t) q(x) \mathrm{d} x}{\int_{\mathbb{R}^{n}} q(x) \mathrm{d} x}=0, \\
\Phi(q, T)=\int_{\mathbb{R}^{n}} x^{*} M_{T} x q(x) \mathrm{d} x,
\end{array}\right.
$$

in which we recall the notation:

$$
U(x, q, t)=\frac{\partial \Phi(q, t)}{\partial q}(x), V(q, t)(x, y)=\frac{\partial^{2} \Phi(q, t)}{\partial q^{2}}(x, y) .
$$

We can next write the Master equation (3.17), which is the equation for $U(x, q, t)$. We get:

$$
\left\{\begin{aligned}
& \frac{\partial U}{\partial t}+\operatorname{tr}\left(a D_{x}^{2} U(x, q, t)\right)+\operatorname{tr}\left(a \int_{\mathbb{R}^{n}} D_{\xi}^{2} V(q, t)(x, \xi) q(\xi) \mathrm{d} \xi\right)+H x \cdot H \int_{\mathbb{R}^{n}} \xi V(q, t)(x, \xi) q(\xi) \mathrm{d} \xi \\
&+\frac{1}{2} \int_{\mathbb{R}^{n}} \int_{\mathbb{R}^{n}} \frac{\partial V(q, t)}{\partial q}(\xi, \eta)(x) H \xi \cdot H \eta q(\xi) q(\eta) \mathrm{d} \xi \mathrm{d} \eta+x^{*} M x+F x \cdot D_{x} U(x, q, t)+\int_{\mathbb{R}^{n}} F \xi \cdot D_{\xi} V(q, t)(x, \xi) q(\xi) \mathrm{d} \xi \\
&+\frac{1}{4} \frac{\int_{\mathbb{R}^{n}} D_{x} U(x, q, t) q(x) \mathrm{d} x \cdot G N^{-1} G^{*} \int_{\mathbb{R}^{n}} D_{x} U(x, q, t) q(x) \mathrm{d} x}{\left(\int_{\mathbb{R}^{n}} q(x) \mathrm{d} x\right)^{2}}-\frac{1}{2} D_{x} U(x, q, t) \cdot G N^{-1} G^{*} \frac{\int_{\mathbb{R}^{n}} D_{\xi} U(\xi, q, t) q(\xi) \mathrm{d} \xi}{\int_{\mathbb{R}^{n}} q(\xi) \mathrm{d} \xi} \\
&-\frac{1}{2} \int_{\mathbb{R}^{n}} D_{\xi} V(q, t)(x, \xi) q(\xi) \mathrm{d} \xi \cdot G N^{-1} G^{*} \frac{\int_{\mathbb{R}^{n}} D_{\xi} U(\xi, q, t) q(\xi) \mathrm{d} \xi}{\int_{\mathbb{R}^{n}} q(\xi) \mathrm{d} \xi}=0 \\
& U(x, q, T)=x^{*} M_{T} x .
\end{aligned}\right.
$$

\subsection{System of HJB-FP equations}

We now write the system of HJB-FP equations (3.22) and (3.24). We look for a pair $(u(x, t), q(x, t))$ the adapted random fields solution of the coupled system:

$$
\begin{aligned}
& \left\{\begin{aligned}
& d_{t} q=\left(\operatorname{tr}\left(a D_{x}^{2} q\right)-\operatorname{div}\left[\left(F x-\frac{1}{2} G N^{-1} G^{*} \frac{\int_{\mathbb{R}^{n}} D_{\xi} u(\xi, t) q(\xi, t) \mathrm{d} \xi}{\int_{\mathbb{R}^{n}} q(\xi, t) \mathrm{d} \xi}\right) q(x, t)\right]\right) \mathrm{d} t+q(x, t) H x \cdot \mathrm{d} z(t), \\
& q(x, 0)=q(x)
\end{aligned}\right. \\
& \left\{\begin{aligned}
-d_{t} u= & \left(\operatorname{tr}\left(a D_{x}^{2} u\right)+x^{*} M x+F x \cdot D_{x} u(x, t)+\frac{1}{4} \frac{\int_{\mathbb{R}^{n}} D_{\xi} u(\xi, t) q(\xi, t) \mathrm{d} \xi \cdot G N^{-1} G^{*} \int_{\mathbb{R}^{n}} D_{\xi} u(\xi, t) q(\xi, t) \mathrm{d} \xi}{\left(\int_{\mathbb{R}^{n}} q(\xi, t) \mathrm{d} \xi\right)^{2}}\right. \\
& \left.-\frac{1}{2} D_{x} u(x, t) \cdot G N^{-1} G^{*} \frac{\int_{\mathbb{R}^{n}} D_{\xi} u(\xi, t) q(\xi, t) \mathrm{d} \xi}{\int_{\mathbb{R}^{n}} q(\xi, t) \mathrm{d} \xi}\right) \mathrm{d} t-K(x, t) \cdot(\mathrm{d} z(t)-H x d t), \\
u(x, T)= & x^{*} M_{T} x .
\end{aligned}\right.
\end{aligned}
$$

The random field $K(x, t)$ can be expressed as:

$$
K(x, t)=H \int_{\mathbb{R}^{n}} \xi V\left(q_{t}, t\right)(x, \xi) q(\xi, t) \mathrm{d} \xi .
$$


The key result is that we can solve this system of equations explicitly and obtain the optimal control. We introduce the matrix $\pi(t)$ solution of the Riccati equation:

$$
\left\{\begin{array}{l}
\frac{\mathrm{d} \pi}{\mathrm{d} t}+\pi F+F^{*} \pi-\pi G N^{-1} G^{*} \pi+M=0 \\
\pi(T)=M_{T}
\end{array}\right.
$$

We next introduce the function $Z(x, \rho, t)$ solution of the deterministic linear PDE:

$$
\left\{\begin{array}{l}
\frac{\partial Z}{\partial t}+D_{x} Z \cdot\left(F-\Gamma(\rho, t) H^{*} H\right) x+D_{\rho} Z \cdot \Phi^{*}(t) H^{*} H(\Phi(t) b(\rho, t)+x)+\frac{1}{2} \operatorname{tr}\left(D_{x}^{2} Z\left(2 a+\Gamma(\rho, t) H^{*} H \Gamma(\rho, t)\right)\right) \\
\quad+\frac{1}{2} \operatorname{tr}\left(D_{\rho}^{2} Z \Phi^{*}(t) H^{*} H \Phi(t)\right)-\operatorname{tr}\left(D_{x \rho}^{2} Z \Phi^{*}(t) H^{*} H \Gamma(\rho, t)\right)+x^{*} \pi(t) G N^{-1} G^{*} \pi(t) x+2 \operatorname{tr}(a \pi(t))=0 \\
Z(x, \rho, T)=0
\end{array}\right.
$$

We next introduce the pair of adapted processes $(\hat{x}(t), \rho(t))$ the solution of the system of SDE:

$$
\begin{gathered}
\left\{\begin{array}{l}
\mathrm{d} \hat{x}=\left(F-G N^{-1} G^{*} \pi(t)\right) \hat{x}(t) \mathrm{d} t+\Gamma(\rho(t), t) H^{*}(\mathrm{~d} z(t)-H \hat{x}(t) \mathrm{d} t), \\
\hat{x}(0)=\bar{x}_{0}
\end{array}\right. \\
\qquad\left\{\begin{array}{l}
\mathrm{d} \rho=\Phi^{*}(t) H^{*}(\mathrm{~d} z(t)-H(\hat{x}(t)-b(\rho(t), t))), \\
\rho(0)=0 .
\end{array}\right.
\end{gathered}
$$

They are built on a convenient probablity space on which $z(t)$ is a standard Wiener process with values in $\mathbb{R}^{d}$. We associate to the pair $(\hat{x}(t), \rho(t))$ the unnormalized conditional probability $q(x, t)$ defined by Zakai equation:

$$
\left\{\begin{array}{l}
d_{t} q=\left(\operatorname{tr}\left(a D_{x}^{2} q\right)-\operatorname{div}\left[\left(F x-G N^{-1} G^{*} \pi(t) \hat{x}(t)\right) q(x, t)\right]\right) \mathrm{d} t+q(x, t) H x \cdot \mathrm{d} z(t), \\
q(x, 0)=q(x) .
\end{array}\right.
$$

We next define the random field

$$
u(x, t):=x^{*} \pi(t) x+Z(x-\hat{x}(t), \rho(t), t) .
$$

We now state the main result of our paper:

Theorem 5.1. We have the property:

$$
\int_{\mathbb{R}^{n}} D_{x} Z(x-\hat{x}(t), \rho(t), t) q(x, t) \mathrm{d} x=0, \text { a.s., for any } t,
$$

and $u(x, t)$ and $q(x, t)$ defined by (5.18) and (5.17), respectively, are solutions of (5.10) and (5.11). The optimal control is given by

$$
\hat{v}(t)=\hat{v}\left(q_{t}, u_{t}\right)=-N^{-1} G^{*} \pi(t) \hat{x}(t) .
$$


Proof. We first prove (5.19). We differentiate (5.14) with respect to $x$ in order to obtain:

$$
\left\{\begin{array}{l}
\frac{\partial}{\partial t} D_{x} Z+D_{x}^{2} Z\left(F-\Gamma(\rho, t) H^{*} H\right) x+\left(F^{*}-H^{*} H \Gamma(\rho, t)\right) D_{x} Z+D_{\rho x}^{2} Z \Phi^{*}(t) H^{*} H(\Phi(t) b(\rho, t)+x) \\
\quad+H^{*} H \Phi(t) D_{\rho} Z+\frac{1}{2} \operatorname{tr}\left(D_{x}^{2} D_{x} Z\left(2 a+\Gamma(\rho, t) H^{*} H \Gamma(\rho, t)\right)\right)+\frac{1}{2} \operatorname{tr}\left(D_{\rho}^{2} D_{x} Z \Phi^{*}(t) H^{*} H \Phi(t)\right) \\
\quad-\operatorname{tr}\left(D_{x \rho}^{2} D_{x} Z \Phi^{*}(t) H^{*} H \Gamma(\rho, t)\right)+2 \pi(t) G N^{-1} G^{*} \pi(t) x=0 \\
Z(x, \rho, T)=0 .
\end{array}\right.
$$

We next consider $q(x, t)$ defined by (5.17). A long calculation then shows that:

$$
\left\{\begin{array}{l}
d_{t} \int_{\mathbb{R}^{n}} D_{x} Z(x-\hat{x}(t), \rho(t), t) q(x, t) \mathrm{d} x=-\left(F^{*}-H^{*} H \Gamma(\rho(t), t)+H^{*} H \Phi(t)\right) \int_{\mathbb{R}^{n}} D_{x} Z(x-\hat{x}(t), \rho(t), t) q(x, t) \mathrm{d} x \mathrm{~d} t \\
+\left(\int_{\mathbb{R}^{n}}\left[-D_{x}^{2} Z(x-\hat{x}(t), \rho(t), t) \Gamma(\rho(t), t)+D_{x \rho}^{2} Z(x-\hat{x}(t), \rho(t), t) \Phi^{*}(t)+D_{x} Z(x-\hat{x}(t), \rho(t), t) x^{*}\right] q(x, t)\right) H^{*} \mathrm{~d} z(t), \\
\int_{\mathbb{R}^{n}} D_{x} Z(x-\hat{x}(T), \rho(T), T) q(x, T) \mathrm{d} x=0 .
\end{array}\right.
$$

From this relation, it follows that

$$
\int_{\mathbb{R}^{n}} D_{x} Z(x-\hat{x}(t), \rho(t), t) q(x, t) \mathrm{d} x \cdot \exp \left(\int_{0}^{t}\left(F^{*}-H^{*} H \Gamma(\rho(s), s)+H^{*} H \Phi(s)\right) \mathrm{d} s\right)
$$

is a $\mathcal{Z}^{t}$ martingale. Since it vanishes at $T$, it is 0 at any $t$, a.e. Hence $(5.19)$ is obtained. Consider $u(x, t)$ by formula (5.18), therefore, using (4.18) and (5.19), we get:

$$
\frac{\int_{\mathbb{R}^{n}} D_{\xi} u(\xi, t) q(\xi, t) \mathrm{d} \xi}{\int_{\mathbb{R}^{n}} q(\xi, t) \mathrm{d} \xi}=2 \pi(t) \hat{x}(t) .
$$

To check (5.10) we have to check:

$$
\left\{\begin{aligned}
-d_{t} u= & {\left[\operatorname{tr}\left(a D_{x}^{2} u\right)+x^{*} M x+F x \cdot D_{x} u+\hat{x}(t)^{*} \pi(t) G N^{-1} G^{*} \pi(t) \hat{x}(t)\right.} \\
& \left.-D_{x} u \cdot G N^{-1} G^{*} \pi(t) \hat{x}(t)\right] \mathrm{d} t-K(x, t) \cdot(\mathrm{d} z(t)-H x d t), \\
u(x, T)= & x^{*} M_{T} x .
\end{aligned}\right.
$$

Note that the final condition can be trivially verified. We can check (5.24) by direct calculation. We obtain also the value of $K(x, t)$ :

$$
K(x, t)=H\left[-\Gamma(\rho(t), t) D_{x} Z(x-\hat{x}(t), \rho(t), t)+\Phi(t) D_{\rho} Z(x-\hat{x}(t), \rho(t), t)\right] .
$$

So we have proven that $(u(x, t), q(x, t))$ is the solution of the system of HJB-FP equations (5.10) and (5.11). The result (5.20) is an immediate consequence of (5.23). The proof is complete.

\subsection{Complements}

The result (5.20) is important. It shows that the optimal control of the problem (5.2) and (5.3) follows the celebrated "Separation Principle". We recall that in the deterministic case, the optimal control, which is necessarily an open-loop control can be obtained by a linear feedback on the state. Open loop and feedback 
controls are equivalent. The separation principle claims that in the partially observable case, the optimal open loop control (adapted to the observation process) can be obtained by the same feedback as in the deterministic case, replacing the nonobservable state by its best estimate, the Kalman filter. The fact that the separation principle holds is well-known when the initial state follows a Gaussian distribution. We have proven that it holds in general. What drives the separation principle is the linearity of the dynamics and of the observation and the fact that the cost is quadratic. The Gaussian assumption does not play any role. A significant simplification occurs in the Gaussian case, regarding the computation of the Kalman filter. In the Gaussian case, the Kalman filter solves a single equation. In general, the Kalman filter is coupled to another sufficient statistics $\rho(t)$ and the pair $(\hat{x}(t), \rho(t))$ must be obtained simultaneously.

We proceed on by obtaining the value function $\Phi(q, t)$. In fact, we obtain $\Phi(q, 0)$. The same procedure must be repeated at any time $t$. First, we have:

$$
\frac{\partial \Phi(q, 0)}{\partial q}(x)=u(x, 0)=x^{*} \pi(0) x+Z\left(x-\frac{\int_{\mathbb{R}^{n}} \xi q(\xi) \mathrm{d} \xi}{\int_{\mathbb{R}^{n}} q(\xi) \mathrm{d} \xi}, 0,0\right) .
$$

We next obtain $\frac{\partial \Phi}{\partial t}(q, 0)$ by formula $(3.27)$. We obtain:

$$
\begin{aligned}
& \frac{\partial \Phi}{\partial t}(q, 0) \\
= & -\operatorname{tr}\left(a \int_{\mathbb{R}^{n}} D_{x}^{2} u(x, 0) q(x) \mathrm{d} x\right)-\frac{1}{2} \int_{\mathbb{R}^{n}} H x \cdot K(x, 0) q(x) \mathrm{d} x-\int_{\mathbb{R}^{n}}\left[x^{*} M x+\hat{v}(0)^{*} N \hat{v}(0)+D_{x} u(x, 0) \cdot(F x+G \hat{v}(0))\right] q(x) \mathrm{d} x \\
= & -2 \operatorname{tr}(a \pi(0))-\operatorname{tr}\left(a \int_{\mathbb{R}^{n}} D_{x}^{2} Z\left(x-\bar{x}_{0}, 0,0\right) q(x) \mathrm{d} x\right)-\frac{1}{2} \int_{\mathbb{R}^{n}} H x \cdot H\left(-\Gamma(0,0) D_{x} Z\left(x-\bar{x}_{0}, 0,0\right)+D_{\rho} Z\left(x-\bar{x}_{0}, 0,0\right)\right) q(x) \mathrm{d} x \\
& -\int_{\mathbb{R}^{n}} F x \cdot D_{x} Z\left(x-\bar{x}_{0}, 0,0\right) q(x) \mathrm{d} x+\left[\bar{x}_{0}^{*} \pi^{\prime}(0) \bar{x}_{0}-\operatorname{tr}(\Gamma(0,0)(M+2 \pi(0) F))\right] \int_{\mathbb{R}^{n}} q(x) \mathrm{d} x,
\end{aligned}
$$

where

$$
\bar{x}_{0}=\frac{\int_{\mathbb{R}^{n}} x q(x) \mathrm{d} x}{\int_{\mathbb{R}^{n}} q(x) \mathrm{d} x} .
$$

We can finally obtain the value of $\Phi(q, 0)$. Since we know the optimal control for the problem (5.2) and (5.3), we have:

$$
\Phi(q, 0)=J(\hat{v}(\cdot))=\mathbb{E}\left[\int_{0}^{T} \int_{\mathbb{R}^{n}} q(x, t)\left(x^{*} M x+\hat{x}(t)^{*} \pi(t) G N^{-1} G^{*} \pi(t) \hat{x}(t)\right) \mathrm{d} t+\int_{\mathbb{R}^{n}} q(x, T) x^{*} M_{T} x \mathrm{~d} x\right],
$$

where $q(x, t)$ is the solution of (5.17). Therefore also,

$$
\begin{aligned}
\Phi(q, 0)=\mathbb{E} & {\left[\int_{0}^{T} \nu(t)\left[\hat{x}(t)^{*}\left(M+\pi(t) G N^{-1} G^{*} \pi(t)\right) \hat{x}(t)+\operatorname{tr} M \Gamma(\rho(t), t)\right] \mathrm{d} t\right] } \\
& +\mathbb{E}\left[\nu(T)\left(\hat{x}(T)^{*} M_{T} \hat{x}(T)+\operatorname{tr} M \Gamma(\rho T), T\right)\right] .
\end{aligned}
$$

The triple $(\hat{x}(t), \rho(t), \nu(t))$ is the solution of the system of $\operatorname{SDE}(5.15)$ and (5.16), and

$$
\left\{\begin{array}{l}
\mathrm{d} \nu(t)=\nu(t) H \hat{x}(t) \cdot \mathrm{d} z(t) \\
\nu(0)=\int_{\mathbb{R}^{n}} q(x) \mathrm{d} x
\end{array}\right.
$$


respectively. From the probabilistic formula (5.28), it is easy to derive an analytic formula as follows:

$$
\Phi(q, 0)=\left(\bar{x}_{0}^{*} \pi(0) \bar{x}_{0}+\mu(0,0)\right) \int_{\mathbb{R}^{n}} q(x) \mathrm{d} x,
$$

where $\mu(\rho, t)$ is the solution of the linear PDE:

$$
\left\{\begin{array}{l}
\frac{\partial \mu}{\partial t}+D_{\rho} \mu \cdot \Phi^{*}(t) H^{*} H \Phi(t) b(\rho, t)+\frac{1}{2} \operatorname{tr}\left(D_{\rho}^{2} \mu \Phi^{*}(t) H^{*} H \Phi(t)\right)+\operatorname{tr}\left(\left(M+\pi(t) \Gamma(\rho, t) H^{*} H\right) \Gamma(\rho, t)\right)=0, \\
\mu(\rho, T)=\operatorname{tr}\left(M_{T} \Gamma(\rho, T)\right) .
\end{array}\right.
$$

We can apply these results in the Gaussian case. We first solve the PDEs (5.14) and (5.31). We recall that $\Gamma(\rho, t)=P(t)$, then $Z(x, \rho, t)$ and $\mu(\rho, t)$ are independent of $\rho$ and are easily seen:

$$
Z(x, \rho, t)=x^{*} \Lambda(t) x+\beta(t),
$$

with

$$
\begin{gathered}
\left\{\begin{array}{l}
\frac{d \Lambda}{\mathrm{d} t}+\Lambda(t)\left(F-P(t) H^{*} H\right)+\left(F^{*}-H^{*} H P(t)\right) \Lambda(t)+\pi(t) G N^{-1} G^{*} \pi(t)=0, \\
\Lambda(T)=0,
\end{array}\right. \\
\beta(t)=\int_{t}^{T} \operatorname{tr}\left(\Lambda(s)\left(2 a+P(s) H^{*} H P(s)\right)\right) \mathrm{d} s .
\end{gathered}
$$

Similarly, $\mu(\rho, t)=\mu(t)$ is given by:

$$
\mu(t)=\int_{t}^{T} \operatorname{tr}\left(\pi(s) P(s) H^{*} H P(s)\right) \mathrm{d} s+\operatorname{tr}\left(M_{T} P(T)\right) .
$$

Then,

$$
\left\{\begin{array}{l}
\frac{\partial \Phi(q, 0)}{\partial q}(x)=x^{*} \pi(0) x+\left(x-\bar{x}_{0}\right)^{*} \Lambda(0)\left(x-\bar{x}_{0}\right)+\beta(0), \\
\frac{\partial \Phi}{\partial t}(q, 0)=-2 \operatorname{tr}((a+P(0) F)(\pi(0)+\Lambda(0)))+\bar{x}_{0}^{*} \pi^{\prime}(0) \bar{x}_{0}-\operatorname{tr}(P(0) M)+\operatorname{tr}\left(P(0) H^{*} H P(0) \Lambda(0)\right), \\
\Phi(q, 0)=\bar{x}_{0}^{*} \pi(0) \bar{x}_{0}+\mu(0) .
\end{array}\right.
$$

\section{Comparison}

\subsection{Objectives}

We compare in this section our work with another recent approach of Bandini et al. [2]. They consider a general problem of stochastic control with partial information, to which our problem can be reduced. Their set up leads to a conditional probability, (hence normalized) solution of a linear stochastic PDE, which they call DMZ equation (for Duncan-Mortensen-Zakai equation). They formulate a control problem for this infinite dimensional state equation, for which they write a Bellman equation. The solution is a functional on the Wasserstein space of probability measures, since indeed the state is a probability. When we formulate our problem in their set up, our Zakai equation cannot be their DMZ equation, since we do not have a probability, but an unnormalized 
probability. To make the comparison easy we keep our model, but we follow the setup of [2]. We explain the difference between the two equations, and also the difference between our own Bellman equation and their Bellman equation. Although our problem can appear as a particular case of [2], it is at the price of complicating it, which turns out to be not suitable. The discussion will explain the reasons. [2] provides an example with linear equations, which does not cover ours. In the set up of [2], our system remains nonlinear, which is also a consequence of the complication of the approach. We remain formal in our presentation, since we want to discuss the concepts and compare the methods.

\subsection{Use of the set up of [2]}

In the set up of [2], we consider the pair $(x(t), \eta(t))$ the solution of the system:

$$
\begin{gathered}
\left\{\begin{array}{l}
\mathrm{d} x=g(x, v) \mathrm{d} t+\sigma(x) \mathrm{d} w \\
x(0)=x_{0}
\end{array}\right. \\
\left\{\begin{array}{l}
\mathrm{d} \eta(t)=\eta(t) h(x(t)) \cdot \mathrm{d} z(t), \\
\eta(0)=\eta_{0}
\end{array}\right.
\end{gathered}
$$

in which $w(\cdot)$ and $z(\cdot)$ are independent Wiener processes and $x_{0}$ and $\eta_{0}$ are random variables independent of $w(\cdot)$ and $z(\cdot)$. We observe only the process $z(\cdot)$. The DMZ equation introduced by [2] is the equation for the conditional probability of the pair $(x(t), \eta(t))$ given the $\sigma$-algebra $\mathcal{Z}^{t}=\sigma(z(s), s \leq t)$. In (6.1), the control $v(t)$ is simply adapted to $\mathcal{Z}^{t}$.

If $\varphi(\eta, x, t)$ is a deterministic function on $\mathbb{R}^{n+1} \times \mathbb{R}^{+}$, we are interested in the process $\rho(\varphi)(t)=$ $\mathbb{E}\left[\varphi(\eta(t), x(t), t) \mid \mathcal{Z}^{t}\right]$. It is the solution of the DMZ equation. We use the notation:

$$
A_{x} \varphi(\eta, x, t)=-\operatorname{tr}\left(a(x) D_{x}^{2} \varphi(\eta, x, t)\right)
$$

with $a(x)=\frac{1}{2} \sigma(x) \sigma^{*}(x)$. We note

$$
A_{x}^{*} \varphi(\eta, x, t)=-\sum_{i j} \frac{\partial^{2}}{\partial x_{i} \partial x_{j}}\left(a_{i j}(x) \varphi(\eta, x, t)\right)
$$

We next define the operators:

$$
\begin{aligned}
\mathcal{L}^{v(\cdot)} \varphi(\eta, x, t) & =D_{x} \varphi(\eta, x, t) \cdot g(x, v(t))-A_{x} \varphi(\eta, x, t)+\frac{1}{2} \eta^{2}|h(x)|^{2} \frac{\partial^{2} \varphi(\eta, x, t)}{\partial \eta^{2}} \\
\mathcal{M} \varphi(\eta, x, t) & =\eta \frac{\partial \varphi(\eta, x, t)}{\partial \eta} h(x)
\end{aligned}
$$

Then the DMZ equation is:

$$
\left\{\begin{array}{l}
\mathrm{d} \rho(\varphi)(t)=\rho\left(\frac{\partial \varphi}{\partial t}+\mathcal{L}^{v(\cdot)} \varphi\right)(t) \mathrm{d} t+\rho(\mathcal{M} \varphi)(t) \cdot \mathrm{d} z(t) \\
\rho(\varphi)(0)=\mathbb{E} \varphi\left(\eta_{0}, x_{0}, 0\right)
\end{array}\right.
$$


In the sequel, we assume the existence of a density $p(\eta, x, t)$ which is the joint conditional probability density of $(\eta(t), x(t))$ given $\mathcal{Z}^{t}$. It is defined by:

$$
\rho(\varphi)(t)=\int p(\eta, x, t) \varphi(\eta, x, t) \mathrm{d} \eta \mathrm{d} x
$$

The conditional probability density is the solution of the stochastic PDE:

$$
\left\{\begin{array}{l}
\mathrm{d} p+\left[A_{x}^{*} p(\eta, x, t)+\operatorname{div}(g(x, v(t)) p(\eta, x, t))-\frac{1}{2}|h(x)|^{2} \frac{\partial^{2}}{\partial \eta^{2}}\left(\eta^{2} p(\eta, x, t)\right)\right] \mathrm{d} t=-h(x) \frac{\partial}{\partial \eta}(\eta p(\eta, x, t)) \cdot \mathrm{d} z(t) \\
p(\eta, x, 0)=p_{0}(\eta, x)
\end{array}\right.
$$

It is easy to check that

$$
q(x, t)=\int \eta p(\eta, x, t) \mathrm{d} \eta
$$

is the solution of Zakai equation (2.9), provided that the initial condition

$$
q_{0}(x)=\int \eta p_{0}(\eta, x) \mathrm{d} \eta
$$

It is then clear that although $p(\eta, x, t)$ is indeed a probability density, while $q(x, t)$ is not. Conversely, if we start with $q_{0}(x)$ and want to solve Zakai equation (2.9), we can use (6.10) by looking for $p(\eta, x, t)$ the solution of the DMZ equation (6.9). We need to take the initial condition of product measure:

$$
p_{0}(\eta, x)=\delta\left(\eta-\int q_{0}(\xi) \mathrm{d} \xi\right) \otimes \frac{q_{0}(x)}{\int q_{0}(\xi) \mathrm{d} \xi}
$$

This is not a probability density, so we need to use the weak formulation, to proceed.

We get some kind of interesting quandary. Using the set up [2] we can use probability measures, the Wasserstein topology and the lifting method of P.L. Lions, but the price to pay is to increase the dimension by 1 , with a nonlinearity. If we stay with the traditional setup, we have to work with unnormalized probability densities. If we can work with densities, it is not a serious drawback, but otherwise we have to find an alternative to the Wasserstein space and the lifting procedure, and it is not clear how to proceed. We can, of course, consider Kushner equation, instead of Zakai equation, whose solution is a probability. But Kushner equation is nonlinear, conversely to Zakai equation.

\subsection{Bellman equation}

We can extend the comparison at the level of Bellman equation. We consider $p^{v(\cdot)}(\eta, x, s), s \geq t$, the solution of

$$
\left\{\begin{array}{l}
\mathrm{d} p+\left[A_{x}^{*} p(\eta, x, s)+\operatorname{div}(g(x, v(s)) p(\eta, x, s))-\frac{1}{2}|h(x)|^{2} \frac{\partial^{2}}{\partial \eta^{2}}\left(\eta^{2} p(\eta, x, s)\right)\right] \mathrm{d} s=-h(x) \frac{\partial}{\partial \eta}(\eta p(\eta, x, s)) \cdot \mathrm{d} z(s), \\
p(\eta, x, t)=p(\eta, x)
\end{array}\right.
$$


where $p(\eta, x)$ is a probability density, denoted in the sequel by $p$. We can then define the payoff $J_{p, t}(v(\cdot))$ by:

$$
J_{p, t}(v(\cdot))=\mathbb{E}\left[\int_{t}^{T} \int p^{v(\cdot)}(\eta, x, s) \eta f(x, v(s)) \mathrm{d} \eta \mathrm{d} x \mathrm{~d} s+\int p^{v(\cdot)}(\eta, x, T) \eta f_{T}(x) \mathrm{d} \eta \mathrm{d} x\right],
$$

and we define the value function

$$
\Psi(p, t)=\inf _{v(\cdot)} J_{p, t}(v(\cdot))
$$

We can write Bellman equation corresponding to this problem; indeed,

$$
\left\{\begin{aligned}
\frac{\partial \Psi}{\partial t}+ & \int\left[-A_{x} \frac{\partial \Psi}{\partial p}(p, t)(\eta, x)+\frac{1}{2}|h(x)|^{2} \eta^{2} \frac{\partial^{2}}{\partial \eta^{2}} \frac{\partial \Psi}{\partial p}(p, t)(\eta, x)\right] p(\eta, x) \mathrm{d} \eta \mathrm{d} x \\
+ & \frac{1}{2} \iint \eta \tilde{\eta} \frac{\partial^{2}}{\partial \eta \partial \tilde{\eta}} \frac{\partial^{2} \Psi}{\partial p^{2}}(p, t)(\eta, x ; \tilde{\eta}, \tilde{x}) h(x) \cdot h(\tilde{x}) p(\eta, x) p(\tilde{\eta}, \tilde{x}) \mathrm{d} \eta \mathrm{d} \tilde{\eta} \mathrm{d} x \mathrm{~d} \tilde{x} \\
& +\inf _{v} \int\left[\eta f(x, v)+D_{x} \frac{\partial \Psi}{\partial p}(p, t)(\eta, x) \cdot g(x, v)\right] p(\eta, x) \mathrm{d} \eta \mathrm{d} x=0, \\
\Psi(p, T) & =\int \eta f_{T}(x) p(\eta, x) \mathrm{d} \eta \mathrm{d} x
\end{aligned}\right.
$$

If we compare with the Bellman equation (3.11) rewritten with the current notation (with argument an unnormalized probability), we obtain:

$$
\left\{\begin{array}{l}
\frac{\partial \Phi}{\partial t}+\int\left(-A_{x} \frac{\partial \Phi}{\partial q}(q, t)(x) q(x)\right) \mathrm{d} x+\frac{1}{2} \iint \frac{\partial^{2} \Phi}{\partial q^{2}}(q, t)(x ; \tilde{x}) h(x) \cdot h(\tilde{x}) q(x) q(\tilde{x}) \mathrm{d} x \mathrm{~d} \tilde{x} \\
\quad+\inf _{v} \int\left[f(x, v)+D_{x} \frac{\partial \Phi}{\partial q}(q, t)(x) \cdot g(x, v)\right] q(x) \mathrm{d} x=0, \\
\Phi(q, T)=\int f_{T}(x) q(x) \mathrm{d} x .
\end{array}\right.
$$

Equations (6.16) and (6.17) are linked by the formulas:

$$
\begin{aligned}
& \Psi(p, t)=\Phi\left(\int \eta p(\eta, \cdot) \mathrm{d} \eta, t\right) \\
& \Phi(q, t)=\Psi\left(\delta\left(\cdot-\int q(\xi) \mathrm{d} \xi\right) \otimes \frac{q(\cdot)}{\int q(\xi) \mathrm{d} \xi}, t\right) .
\end{aligned}
$$

\subsection{The linear case}

If we go back to the linear case (4.3), we get:

$$
\left\{\begin{array}{l}
\mathrm{d} x=(F x+G v) \mathrm{d} t+\sigma \mathrm{d} w \\
\mathrm{~d} \eta=\eta H x \cdot \mathrm{d} z
\end{array}\right.
$$

therefore, in the setup [2], we still have a nonlinear system. Therefore, we cannot use the linear case of [2]. This explains why our formulas are completely different. The fact that we have an explicit solution of the system 
of HJB-FP equations does not imply that we have an explicit solution of Bellman equation. This is consistent with the spirit of the method of characteristics.

\section{REFERENCES}

[1] E. Bandini, A. Calvia and K. Colaneri, Stochastic filtering of a pure jump process with predictable jumps and path-dependent local characteristics. Preprint arXiv: 2004.12944 (2020).

[2] E. Bandini, A. Cosso, M. Fuhrman and H. Pham, Randomized filtering and bellman equation in Wasserstein space for partial observation control problem. Stoch. Process. Appl. 129 (2019) 674-711.

[3] A. Bensoussan, Stochastic Control of Partially Observable Systems. Cambridge University Press (1992).

[4] A. Bensoussan, J. Frehse and P. Yam, Vol. 101 of Mean Field Games and Mean Field Type Control Theory. Springer, New York (2013).

[5] R. Buckdahn, J. Li and J. Ma, A mean-field stochastic control problem with partial observations. Ann. Appl. Probab. 27 (2017) 3201-3245.

[6] A. Calvia, Stochastic filtering and optimal control of pure jump Markov processes with noise-free partial observation. ESAIM: COCV 26 (2020) 25.

[7] P. Cardaliaguet, F. Delarue, J.M. Lasry and P.L. Lions, The Master Equation and the Convergence Problem in Mean Field Games. Preprint arXiv: 1509.02505 (2015).

[8] M.H.M. Chau, Y. Lai and S.C.P. Yam, Discrete-time mean field partially observable controlled systems subject to common noise. Appl. Math. Optim. 76 (2017) 59-91.

[9] D. Firoozi and P.E. Caines, $\epsilon$-Nash Equilibria for major minor LQG mean field games with partial observations of all agents. IEEE Trans. Autom. Control 66 (2021) 2778-2786.

[10] F. Gozzi and A. Świech, Hamilton-Jacobi-Bellman equations for the optimal control of the Duncan-Mortensen-Zakai equation. J. Funct. Anal. 172 (2000) 466-510.

[11] P.L. Lions, Viscosity solutions of fully nonlinear second-order equations and optimal stochastic control in infinite dimensions. Part I: The case of bounded stochastic evolutions. Acta Math. 161 (1988) 243-278.

[12] A.M. Makowski, Filtering formulae for partially observed linear systems with non-Gaussian initial conditions. Stochastics $\mathbf{1 6}$ (1986) 1-24.

[13] M. Nisio, Vol. 72 of Stochastic control theory: Dynamic programming principle. Springer (2014).

[14] N. Saldi, T. Başar and M. Raginsky, Partially-observed discrete-time risk-sensitive mean-field games. In 2019 IEEE 58th Conference on Decision and Control (CDC). IEEE (2019) 317-322.

[15] N. Sen and P.E. Caines, Mean field game theory for agents with individual-state partial observations. In 2016 IEEE 55th Conference on Decision and Control (CDC). IEEE (2016) 6105-6110.

[16] S.G. Subramanian, M.E. Taylor, M. Crowley and P. Poupart, Partially Observable Mean Field Reinforcement Learning. Preprint arXiv: 2012.15791 (2020).

[17] S. Tang, The maximum principle for partially observed optimal control of stochastic differential equations. SIAM J. Control Optim. 36 (1998) 1596-1617. 